Article

\title{
An Oral FMT Capsule as Efficient as an Enema for Microbiota Reconstruction Following Disruption by Antibiotics, as Assessed in an In Vitro Human Gut Model
}

\author{
Cécile Verdier 1,2,t, Sylvain Denis ${ }^{1,+}$, Cyrielle Gasc ${ }^{2}$, Lilia Boucinha ${ }^{2}$, Ophélie Uriot ${ }^{1}$, Dominique Delmas ${ }^{2}$, \\ Joël Dore ${ }^{2,3}$, Corentin Le Camus ${ }^{2}$, Carole Schwintner ${ }^{2,+}$ and Stéphanie Blanquet-Diot ${ }^{1, \neq, *}$ \\ 1 UMR 454 MEDIS, Université Clermont Auvergne, INRAe, F-63000 Clermont-Ferrand, France; \\ cecile.verdier@uca.fr (C.V.); sylvain.denis@uca.fr (S.D.); ophelie.uriot@uca.fr (O.U.) \\ 2 MaaT Pharma, F-69007 Lyon, France; cgasc@maat-pharma.com (C.G.); lboucinha@maat-pharma.com (L.B.); \\ dominique.delmas@valotec.com (D.D.); joel.dore@inrae.fr (J.D.); clecamus@maat-pharma.com (C.L.C.); \\ cschwintner@maat-pharma.com (C.S.) \\ 3 MICALIS and MétaGénoPolis, Université Paris Saclay, INRAe, AgroParisTech, \\ F-78350 Jouy-en-Josas, France \\ + Co-first authors. \\ Citation: Verdier, C.; Denis, S.; \\ $\ddagger$ Co-senior authors. \\ * Correspondence: stephanie.blanquet@uca.fr
}

Delmas, D.; Dore, J.; Camus, C.L.;

Schwintner, C.; Blanquet-Diot, S.

An Oral FMT Capsule as Efficient as

an Enema for Microbiota

Reconstruction Following

Disruption by Antibiotics, as

Assessed in an In Vitro Human Gut

Model. Microorganisms 2021, 9, 358.

https://doi.org/10.3390/

microorganisms 9020358

Academic Editor: Peter C. Konturek

Received: 12 January 2021

Accepted: 9 February 2021

Published: 11 February 2021

Publisher's Note: MDPI stays neutral with regard to jurisdictional

claims in published maps and institutional affiliations.

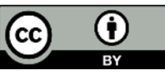

Copyright: $@ 2021$ by the authors. Licensee MDPI, Basel, Switzerland. This article is an open access article distributed under the terms and conditions of the Creative Commons Attribution (CC BY) license (http://creativecommons.org/licenses/by/4.0/).

\begin{abstract}
Fecal microbiota transplantation (FMT) is an innovative therapy already used in humans to treat Clostridioides difficile infections associated with massive use of antibiotics. Clinical studies are obviously the gold standard to evaluate FMT efficiency but remain limited by regulatory, ethics, and cost constraints. In the present study, an in vitro model of the human colon reproducing medically relevant perturbation of the colonic ecosystem by antibiotherapy was used to compare the efficiency of traditional FMT enema formulations and a new oral capsule in restoring gut microbiota composition and activity. Loss of microbial diversity, shift in bacterial populations, and sharp decrease in fermentation activities induced in vivo by antibiotherapy were efficiently reproduced in the in vitro model, while capturing inter-individual variability of gut microbiome. Oral capsule was as efficient as enema to decrease the number of disturbed days and bacterial load had no effect on enema performance. This study shows the relevance of human colon models as an alternative approach to in vivo assays during preclinical studies for evaluating FMT efficiency. The potential of this in vitro approach could be extended to FMT testing in the management of many digestive or extra-intestinal pathologies where gut microbial dysbiosis has been evidenced such as inflammatory bowel diseases, obesity or cancers.
\end{abstract}

Keywords: gut microbiota; antibiotic; dysbiosis; FMT; enema; oral capsule; in vitro gut model

\section{Introduction}

The digestive tract harbors the largest and most complex microbial community of the human body, namely the gut microbiota, which is mainly composed of thousands of bacterial species but also members of Archaea, Eukaryotes, and viruses [1-3]. The highest bacterial density is found in the large intestine with up to $10^{12}$ cells per gram of intestinal content [4]. The gut microbiota plays a fundamental role for the host under normal homeostasis, and is involved in physiological, nutritional, and immunological processes [4]. A large number of studies in animal models and humans have shown that a persistent imbalance in gut microbial community is associated with intestinal disorders such as inflammatory bowel diseases or irritable bowel syndrome or even extra-digestive pathologies like diabetes, obesity, cancers or neurological disorders. This alteration, named dysbiosis, 
has been associated with loss of richness and diversity, loss of keystone taxa, shift in metabolic pathways and/or bloom of pathobionts like Enterobacteriaceae or Clostridiaceae [4,5]. This raises a number of questions such as the causal relationship between disorder and gut microbiota alteration or if a return to an equilibrium state would be sufficient to eradicate or alleviate symptoms and/or how to come back to a "healthy" state.

Fecal microbiota transplantation (FMT) is the administration of fecal material from a healthy donor into the intestinal tract of a patient. FMT is performed in order to restore an altered microbiome, get back to a healthy state and thus confer a health benefit. FMT was reported to be used in traditional Chinese medicine 1700 years ago as a "yellow soup" to treat severe diarrhea $[6,7]$ and its first description in English literature was recorded in 1958 to treat pseudomembranous colitis [8]. To date, FMT is a successful treatment option for patients with recurrent or refractory Clostridioides difficile infection (rCDI), a major cause of antibiotic-associated diarrhea $[9,10]$. However, FMT has also promising therapeutic value in several other disorders associated with gut microbial dysbiosis such as inflammatory bowel diseases, irritable bowel syndrome, cancers, acute myeloid leukemia, graft-versus-host disease, neurodegenerative disorders, autism, obesity and others $[4,10-$ 12]. FMT can be divided into two types: autologous FMT using patient's own feces (stool collection being performed before deleterious treatment) and allogenic FMT with the use of related or unrelated healthy donor fecal samples [13,14]. While autologous FMT has proven its efficiency in restoring intestinal microbiota composition following disruption by antibiotics use during allogeneic hematopoietic stem cell transplantation [15], allogenic FMT was found to be most efficient in case of rCDI [16]. Several routes of FMT administration are available, namely enema, colonoscopy, naso-gastric duodenal, jejunal infusion or oral capsule $[6,7,17]$. Each approach presents some limits such as difficulties to retain administered suspension for enema, risk of vomiting and aspiration as well as discomfort during administration for naso-gastric tubes and required sedation and risk of tissue perforation for jejunal infusion and colonoscopy [18]. Oral capsules were recently developed to address gaps and limitations previously observed in other FMT routes of administration. Oral capsules offer the least invasive, cheapest and most easily stored administered form and eliminate several procedural risks encountered with the other routes of FMT treatment. However, those capsules have been associated with side effects - such as risk of vomiting and aspiration - and sometimes they can fail to reach their intestinal target $[14,17]$. In addition, Kao et al. have shown that oral capsules are non-inferior to delivery by colonoscopy and represent an effective approach in the treatment of rCDI [19].

Obviously, clinical studies remain as the gold standard approach to evaluate the effect of FMT treatment on gut microbial restoration and determine associated health benefits. Nevertheless, this approach may be hampered by heavy regulations, ethical concerns and high experimental cost. In order to reduce human experiments, animal models can be used in preclinical phases to evaluate FMT efficiency. Studies mainly involve conventional mice models but also Dextran Sodium Sulfate-induced colitis mice models or even human microbiota-associated rodent models [20-23]. These models have been advantageously used to assess FMT effect following antibiotic treatment or chemotherapy [20] but also as an alternative strategy in inflammatory bowel disease or in the management of metabolic disorders [21]. In vivo models present the great advantage to integrate host biological responses after FMT treatments such as weight gain/loss, inflammatory response, epithelial barrier integrity or host cell receptor activation. However, this in vivo approach in rodent remains limited by increased ethical and regulatory constraints and also by the high level of expertise required to handle animals (especially human microbiota-associated mice). Furthermore, significant differences in terms of both diet and digestive physiology, including gut microbiota, have been found between animals and humans [24]. FMT is also often administered by force-feeding in rodents which is far from the rectal administration frequently used in clinical practices [20,22].

A relevant alternative to animal experiments in preclinical phases is the use of in vitro models simulating the human digestive environment. Few studies have already used in 
vitro models of the human colon for investigating the effect of FMT on Clostridioides difficile $[25,26]$. Here, we describe for the first time the use of a human colonic system to assess the efficiency of different FMT formulations. For this purpose, we used the wellvalidated ARtificial COLon (ARCOL) system which integrates the main physicochemical and microbial parameters of the human colonic environment based on in vivo data, i.e., $\mathrm{pH}$, temperature, retention time, supply of a nutritive medium reproducing ileal effluent composition, complex and metabolically active colonic microbiota and anaerobiosis maintained by the sole activity of resident microbiota $[27,28]$. Perturbations of gut microbiome were first induced in ARCOL by an antibiotic (ATB) treatment with ciprofloxacin, frequently used in humans to treat a wide range of bacterial infections. Then, the "dysbiotic" in vitro model was used to compare the efficiency of two enema dosages (10 $\mathrm{g}$ and $30 \mathrm{~g}$ ) and a new autologous FMT capsule in restoring gut microbiota composition and metabolic activity (gas and short chain fatty acids-SCFAs-production).

\section{Materials and Methods}

\subsection{Fecal Sampling and FMT Preparation}

Fresh fecal samples were collected from three healthy adult human volunteers (a 27year-old woman and two men aged 35 and 50 years) with no history of antibiotic or probiotic treatment 3 months prior to the beginning of the study. Fecal samples were kept anaerobically for a maximum of $6 \mathrm{~h}$ before treatment. Fecal inocula for the in vitro colon model were prepared using $55 \mathrm{~g}$ of fresh fecal samples under strict anaerobic conditions in a vinyl anaerobic chamber (Coy Laboratory Products Inc, Grass Lake, MI, USA). Stools were mixed with $500 \mathrm{~mL}$ of a $30 \mathrm{mM}$ anaerobic sodium phosphate buffer ( $\mathrm{pH}$ 6.5) supplemented with $1.9 \mathrm{mM}$ cysteine, then the fecal suspension was filtered through a double layer of gauze. The fecal suspension was divided into $100 \mathrm{~mL}$ aliquots that were rapidly transferred into each of the five bioreactors simultaneously inoculated.

Filtrates of the same fecal suspensions were used by MaaT Pharma (Lyon, France) to prepare autologous FMT enema and capsules, according to its manufacturing operating system. FMT enema is a fecal-microbiota suspension for rectal administration stored in a special bag at $-80^{\circ} \mathrm{C}$ (volume of $128-150 \mathrm{~mL}$ for $30 \mathrm{~g}$ enema and $43-50 \mathrm{~mL}$ for $10 \mathrm{~g}$ enema). The $30 \mathrm{~g}$ and $10 \mathrm{~g}$ enemas contain about $30 \times 10^{11}$ and $10 \times 10^{11}$ bacteria, respectively, with a bacterial viability superior to $50 \%$. FMT capsule $(0.45 \mathrm{~g})$ is a caecum-release capsule containing the freeze-dried form of the enema formulation. A capsule contains about $0.35 \times$ $10^{10}$ bacteria, with a bacterial viability superior to $50 \%$. For FMT capsules, only the active ingredient (enema in its freeze-dried form) was introduced into ARCOL model.

\subsection{In Vitro Artificial Colon System ARCOL}

Human colonic conditions were simulated in ARCOL model using MiniBio $500 \mathrm{~mL}$ my-Control bundles and Lucullus ${ }^{\circledR}$ Lite software from Applikon (Delft, The Netherlands). Fermentations were conducted under semi-continuous conditions. The in vitro system reproduces, based on in vivo data, the main physicochemical and microbial conditions encountered in a healthy human adult colon $[27,29,30]$. Briefly, at the beginning of the experiment, fecal suspension $(100 \mathrm{~mL})$ was added into the bioreactor already filled with 200 $\mathrm{mL}$ of nutritive medium while flushing with $\mathrm{O}_{2}$-free $\mathrm{N}_{2}$ gas. Afterwards, anaerobic conditions were maintained exclusively through the sole activity of the resident microbiota and by ensuring the system airtightness. Overproduced gases were collected in a gas sampling bag connected to the condenser. Fermentation temperature was set at $37^{\circ} \mathrm{C}$ and maintained inside the bioreactor using an incorporated panel heater. Colonic $\mathrm{pH}$ and redox potential were constantly recorded (Applikon, Delft, The Netherlands) and $\mathrm{pH}$ was adjusted to a value of 6.3 with an automatic addition of $2 \mathrm{M} \mathrm{NaOH}$. The amount of $\mathrm{NaOH}$ consumed was recorded daily. After one day of batch fermentation, the nutritive medium containing various sources of carbohydrates, proteins, lipids, minerals, and vitamins (Table 1) to closely mimic the composition of human ileal effluents (nutrient availability and 
biliary salts concentration), was continuously introduced into the bioreactor at a flow rate of $0.21 \mathrm{~mL} / \mathrm{min}$. The fermentation medium was stirred at a constant speed of $400 \mathrm{rpm}$. Its volume was monitored using a level sensor and maintained at a constant value of $300 \mathrm{~mL}$ by automatic withdrawal of the fermentation medium, ensuring a mean retention time of $24 \mathrm{~h}$. This set-up with colonic parameters allows a shift from fecal to colonic microbial profiles [31].

Table 1. Composition of the nutritive medium used to feed in vitro ARtificial COLon (ARCOL) system and simulating the composition of human ileal effluents.

\begin{tabular}{|c|c|}
\hline Components & Concentration $(\mathrm{g} / \mathrm{L})$ \\
\hline Potato starch & 5 \\
\hline Corn starch & 2 \\
\hline Cellulose & 1.5 \\
\hline Pectin & 2 \\
\hline Arabinogalactan & 1 \\
\hline Gum arabic & 0.67 \\
\hline Guar gum & 0.33 \\
\hline Inulin & 1 \\
\hline Bacto peptone & 2.5 \\
\hline Vegetable peptone & 2.5 \\
\hline Bacto tryptone & 5 \\
\hline Mucin type II & 4 \\
\hline Yeast extract & 4 \\
\hline Tween 80 & 1 \\
\hline Soy lecithin & 0.375 \\
\hline Egg yolk & 0.125 \\
\hline Bile salts & 0.15 \\
\hline Bile extract & 0.05 \\
\hline $\mathrm{K}_{2} \mathrm{HPO}_{4} 3 \mathrm{H}_{2} \mathrm{O}$ & 1.14 \\
\hline $\mathrm{NaCl}$ & 4.5 \\
\hline $\mathrm{KCl}$ & 4.5 \\
\hline $\mathrm{MgSO}_{4} 7 \mathrm{H}_{2} \mathrm{O}$ & 0.1 \\
\hline $\mathrm{CaCl}_{2} 2 \mathrm{H}_{2} \mathrm{O}$ & 0.03 \\
\hline $\mathrm{FeSO}_{4} 7 \mathrm{H}_{2} \mathrm{O}$ & 0.015 \\
\hline Hemin & 0.005 \\
\hline L-cystein- $\mathrm{HCl}$ & 0.3 \\
\hline $\mathrm{NaHCO}_{3}$ & 0.840 \\
\hline D-Pantothenic acid & $1 \times 10^{-5}$ \\
\hline Nicotinamide & $5 \times 10^{-6}$ \\
\hline 4-aminobenzoic acid & $5 \times 10^{-6}$ \\
\hline Thiamin & $4 \times 10^{-6}$ \\
\hline Menadione & $1 \times 10^{-6}$ \\
\hline D-biotin & $2 \times 10^{-6}$ \\
\hline Vitamin K1 & $1 \times 10^{-6}$ \\
\hline Vitamin B12 & $5 \times 10^{-7}$ \\
\hline $\mathrm{MnSO}_{4} \mathrm{H}_{2} \mathrm{O}$ & $1.7 \times 10^{-4}$ \\
\hline $\mathrm{CoSO}_{4} 7 \mathrm{H}_{2} \mathrm{O}$ & $1.42 \times 10^{-4}$ \\
\hline $\mathrm{ZnSO}_{4} 7 \mathrm{H}_{2} \mathrm{O}$ & $1.44 \times 10^{-4}$ \\
\hline $\mathrm{CuSO}_{4} 5 \mathrm{H}_{2} \mathrm{O}$ & $2.5 \times 10^{-5}$ \\
\hline $\mathrm{NaWO}_{4} 2 \mathrm{H}_{2} \mathrm{O}$ & $3.3 \times 10^{-5}$ \\
\hline $\mathrm{H}_{3} \mathrm{BO}_{3}$ & $6.2 \times 10^{-6}$ \\
\hline
\end{tabular}




$\begin{array}{cl}\mathrm{Na}_{2} \mathrm{MoO}_{4} 2 \mathrm{H}_{2} \mathrm{O} & 2.4 \times 10^{-5} \\ \mathrm{NiCl}_{2} 6 \mathrm{H}_{2} \mathrm{O} & 2.4 \times 10^{-5} \\ \mathrm{Na}_{2} \mathrm{SeO}_{3} & 3.8 \times 10^{-5}\end{array}$

\subsection{Experimental Design of In Vitro Fermentations}

Five bioreactors run in parallel were inoculated with the fecal suspension from one donor (Figure 1) and used as follows: the first bioreactor was used as a control with no antibiotic treatment (control) while the second bioreactor was treated with ciprofloxacin (Sigma-Aldrich, Saint-Louis, MO, USA, 17850-5G-F), with an initial addition of $150 \mathrm{mg}$ on day 6 followed by a continuous supply of $500 \mu \mathrm{g} / \mathrm{mL}$ in the nutritive medium up to day 12 (ATB control). The three other bioreactors were treated with ciprofloxacin as described for ATB control and then received: $27 \mathrm{~mL}$ of enema preparation at day 14 and day 15 (30 g enema), $9 \mathrm{~mL}$ of enema preparation at day 14 and day 15 (10 g enema) or the content of 3 capsules per day for 7 consecutive days from day 14 to day 20 (capsule). The total amount of bacteria administered in ARCOL model with FMT treatment is $1.2 \times 10^{12}, 0.4 \times$ $10^{12}, 1.47 \times 10^{10}$, for $30 \mathrm{~g}$ enema, $10 \mathrm{~g}$ enema and capsule, respectively. Experiments were performed in triplicate (biological replicates named Run1, Run2 and Run3) with fecal samples from each of the three healthy donors. During fermentations, samples were collected daily from the fermentative medium and the atmospheric phase for downstream analyses.

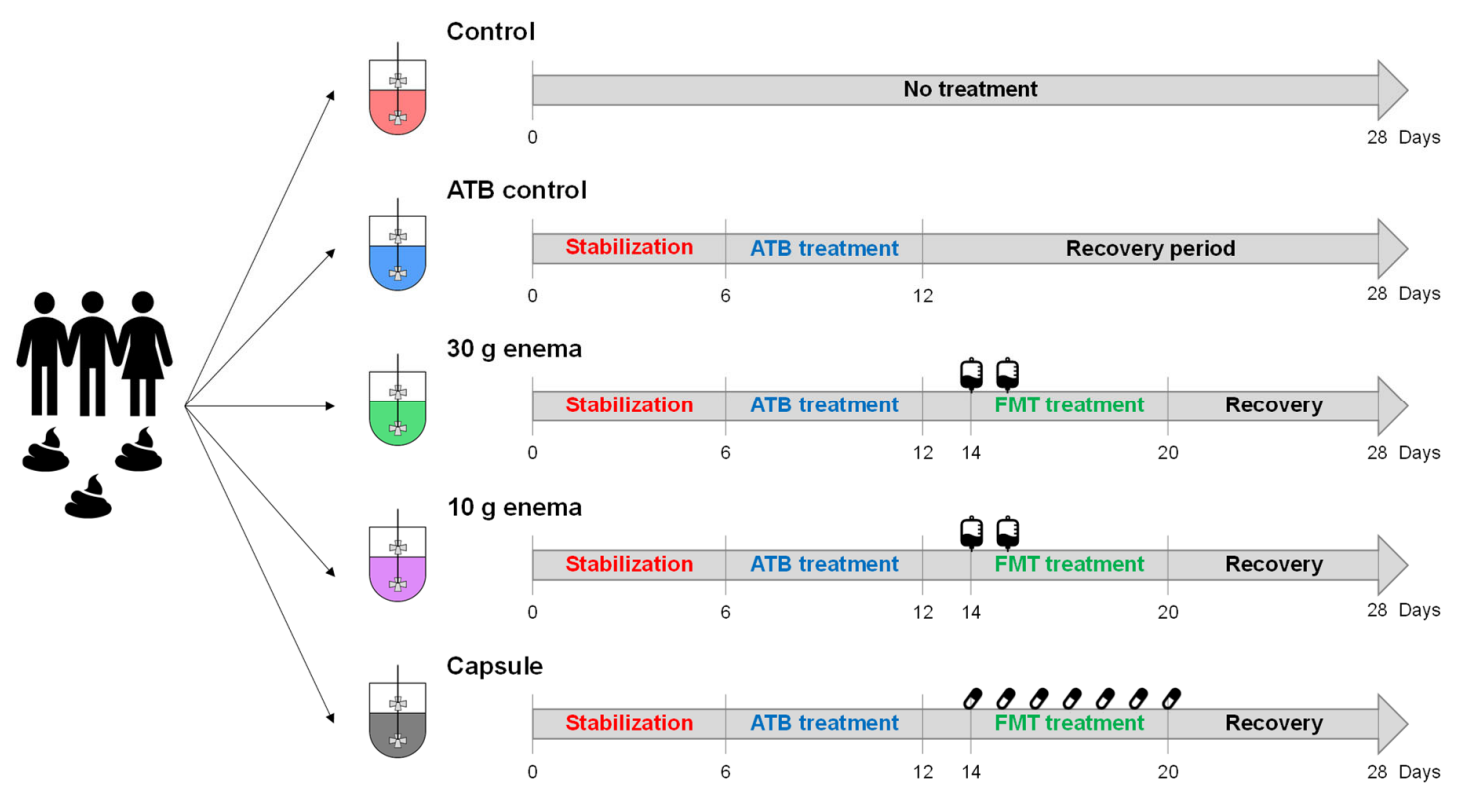

Figure 1. Experimental design of in vitro fermentations in ARtificial COLon (ARCOL) system.

Five bioreactors were inoculated with the same fecal suspension and ran in parallel for 28 days. No treatment was applied in control condition (control, red bioreactor). After a 6-day period of microbiota stabilization, the four other bioreactors were treated with 500 $\mu \mathrm{g} / \mathrm{mL}$ ciprofloxacin for 6 days to induce gut microbiota dysbiosis. Out of the four reactors, one received no FMT treatment (ATB control, blue fermenter). After a 2-day period of antibiotic wash-out, FMT treatment was performed in the three last bioreactors with different modes of administration: $30 \mathrm{~g}$ enema (green bioreactor), $10 \mathrm{~g}$ enema (pink bioreactor) or oral capsule (grey bioreactor). Enema treatments were administered at day 14 and day 15. Oral capsules were administered three times per day for seven days from day 14 to day 20. The recovery period was defined as days of fermentation after cessation of FMT treatment. Experiments were performed in triplicate with feces from three different healthy adult donors (Run1, Run2, and Run3). 


\subsection{Antibiotic Dosage}

Ciprofloxacin concentrations in the fermentative medium were determined using a TurboFlow ${ }^{\mathrm{TM}}$ technology (TLX) coupled to Liquid Chromatography-High Resolution Mass Spectrometry (LC-HRMS, ORBITRAP ${ }^{\circledR}$ technology) at the pharmacological and toxicological analytic unit (CREPTA) of Clermont-Ferrand university hospital, using a method adapted from Hösl et al. 2018 [32] and Lefeuvre et al. 2017 [33].

\subsection{Gut Microbiota Activity}

\subsubsection{Gas}

Analysis of $\mathrm{O}_{2}, \mathrm{~N}_{2}, \mathrm{CO}_{2}, \mathrm{CH}_{4}$, and $\mathrm{H}_{2}$ present in the bioreactor atmospheric phase was performed using a 490 Micro-gas chromatograph (Agilent Technologies, Santa Clara, CA, USA) equipped with two columns, Molecular Sieve 5A, and PoraPlot U, coupled with TCD detectors. Argon was used as the carrier gas. Gas composition was determined using calibration curves made from ambient air $\left(78 \% \mathrm{~N}_{2}, 21 \% \mathrm{O}_{2}, 0.04 \% \mathrm{CO}_{2}\right)$ and two gas mixtures $\mathrm{A}\left(5 \% \mathrm{CO}_{2}, 5 \% \mathrm{H}_{2}, 90 \% \mathrm{~N}_{2}\right)$ and $\mathrm{B}\left(20 \% \mathrm{CO}_{2}, 80 \% \mathrm{H}_{2}\right)$. Results were expressed in relative percentages. Total volume of gases overproduced per day (in $\mathrm{mL}$ ) was also measured by connecting a gas bag to each bioreactor.

\subsubsection{Short Chain Fatty Acids (SCFAs)}

Samples collected from fermentative medium were centrifuged at $18,000 \mathrm{~g}$ for $15 \mathrm{~min}$ at $4{ }^{\circ} \mathrm{C}$ and supernatants were filtered $(0.45 \mu \mathrm{m})$. Concentrations of the three main SCFAs (acetate, propionate and butyrate) were determined using high performance liquid chromatography (HPLC) (Elite LaChrom, HITACHI, San Jose, CA, USA) coupled with a diode-array detector. The HPLC column (Concise Separations, San Jose, CA, USA, ICE-999865 ) and its guard column were maintained at $50{ }^{\circ} \mathrm{C}$. Sulfuric acid $0.008 \mathrm{~N}$ was used as mobile phase and SCFAs were separated at a flow rate of $0.6 \mathrm{~mL} / \mathrm{min}$. Data was analyzed by the EZChrom Elite software at $205 \mathrm{~nm}$. SCFAs concentrations (expressed in $\mathrm{mM}$ or relative percentages) were calculated from standard curves established with known concentrations of acetate, propionate and butyrate $(0,10,25$ and $40 \mathrm{mM})$.

\subsection{Gut Microbiota Composition}

\subsubsection{Flow Cytometry Analysis}

Concentrations of viable bacteria in the fermentation medium were determined by flow cytometry through a live/dead analysis. Samples were 10-fold diluted in sterile physiological water to reach $10^{-4}$ dilution factor. Bacteria were double-stained with the greenfluorescent DNA SYTO 9 dye labelling all bacteria and the red-fluorescent Propidium Iodide dye only penetrating and staining cells with damaged membranes (Molecular probes, Eugene, OR, USA, L34856). Bacterial suspensions were thus incubated for $15 \mathrm{~min}$ at room temperature in the dark with $3.3 \mathrm{mM}$ SYTO 9 and $0.375 \mathrm{mM}$ Propidium Iodide and transferred into BD Trucount ${ }^{\mathrm{TM}}$ Tubes (BD Biosciences, San Jose, CA, USA). Flow cytometry analysis was performed on a BD ${ }^{\mathrm{TM}}$ LSR II cytometer (BD Biosciences, San Jose, CA, USA) and data were collected with BD FACSDiva ${ }^{\mathrm{TM}}$ software. Gating on forwardangle light scatter/side-angle light scatter was used in order to differentiate bacteria from the background. Then, combined red and green fluorescence dot-plots were used to distinguish the various subpopulations. Results were expressed as viable cells per $\mathrm{mL}$ of fermentative medium.

\subsection{2. qPCR Analysis}

Total DNA was extracted from fermentative medium using SmartExtract-DNA Extraction Kit (Eurogentec, Liège, Belgium, SK-DNEX-100), according to manufacturer's instructions. DNA quantity was evaluated with a NanoDrop ${ }^{\text {TM }} 2000$ (Thermo Scientific, Wil- 
mington, DE, USA). Samples were stored at $-20{ }^{\circ} \mathrm{C}$ prior to analysis. Total bacteria concentration was quantified by qPCR analysis performed on a Stratagene Mx3005P apparatus (Agilent, Waldbronn, Germany) using Takyon Low ROX SYBR 2X MasterMix blue dTTP kit (Eurogentec, Liège, Belgium, UF-LSMT-B0701). Each reaction was run in duplicate in a final volume of $10 \mu \mathrm{L}$ with $5 \mu \mathrm{L}$ of Master Mix, $0.45 \mu \mathrm{L}$ of each primer $(10 \mu \mathrm{M})$, $1 \mu \mathrm{L}$ of DNA sample $(10 \mathrm{ng} / \mu \mathrm{L})$, and $3.1 \mu \mathrm{L}$ of ultra-pure water. The following primers targeting 16S rRNA gene were used: BAC338R, 5'- ACTCCTACGGGAGGCAG-3', and BAC516F, 5'-GTATTACCGCGGCTGCTG-3', as described by Yu and colleagues [34]. The amplification conditions consisted in 1 cycle at $95^{\circ} \mathrm{C}$ for $5 \mathrm{~min}$ followed by 40 cycles of 95 ${ }^{\circ} \mathrm{C}$ for $30 \mathrm{~s}, 58^{\circ} \mathrm{C}$ for $30 \mathrm{~s}$, and $72{ }^{\circ} \mathrm{C}$ for $30 \mathrm{~s}$. A final cycle of $5 \mathrm{~min}$ at $95{ }^{\circ} \mathrm{C}$ was included. Standard curves were generated from genomic DNA extracted from ARCOL samples, standardized to $10 \mathrm{ng} / \mu \mathrm{L}$ and serially diluted from $10^{10}$ to $10^{\circ}$ gene copy/mL. Final results were expressed as copy/mL of genomic DNA.

\subsubsection{S rRNA Gene Sequencing and Bioinformatics Analysis}

Genomic DNA was extracted using the NucleoSpin Soil kit (Machery Nagel,Düren, Germany, 740780.50) and samples stored at $-20{ }^{\circ} \mathrm{C}$ before analysis. $16 \mathrm{~S}$ rRNA gene sequencing was performed by Eurofins Genomics (Ebersberg, Germany). A sequencing library targeting the V3-V4 region of the 16S rRNA gene was constructed for each sample using the MyTaq HS-Mix 2X (Bioline, Memphis, TN, USA, BIO-25045) according to manufacturer's instructions. Libraries were then pooled in an equimolar mixture and sequenced in paired-end $(2 \times 300 \mathrm{bp})$ MiSeq V3 runs, Illumina. After amplicon merging using FLASH [35] and quality filtering using Trimmomatic [36], host sequence decontamination was performed with Bowtie2 [37]. Operational Taxonomic Unit (OTU) sequence clustering was performed with an identity threshold of 97\% using VSEARCH [38] and taxonomic profiling was then performed with the Silva SSU database Release 128 [39]. Taxonomic and diversity analyses were performed with $\mathrm{R}$ Statistical Software (R Core Team 2015, version 3.4.4) [40] using vegan and phyloseq packages. For fair comparison, the sequence number of each sample was randomly normalized to the same sequencing depth i.e., 50,000 amplicons per sample and normalized by total bacteria count based on qPCR results. Diversity measures correspond to the median value of 20 subsamplings per sample.

All sequencing data were deposited to the National Center for Biotechnology Information Sequence Read Archive under accession number PRJNA642894.

\subsection{Dysbiosis Criteria}

Criteria selected to determine microbial dysbiosis periods in ARCOL system were based on modifications of both gut microbiota activity and composition compared to stabilized conditions. For microbiota activity, the following parameters were selected: redox potential values, $\mathrm{NaOH}$ consumption, total gas production, $\mathrm{CO}_{2}$ concentration, and SCFA concentrations. Regarding gut microbiota composition, the selected parameters were the following: total viable bacteria as determined by flow cytometry, total bacterial populations measured by qPCR, richness, Shannon and Bray Curtis indexes. In order to establish a dysbiotic period, each day of fermentation from day 6 for a treated bioreactor was compared to day 6 (corresponding to the end of stabilization phase) of the same bioreactor for all $16 \mathrm{~S}$ rRNA gene analysis-related criteria (abundance and diversity indexes). For all other criteria, each day of fermentation from day 6 for a treated bioreactor was compared to the same day of the control bioreactor. All selected criteria and threshold values are summarized in Table 2. 
Table 2. Selected criteria used to determine dysbiosis periods in ARtificial COLon (ARCOL) system.

\begin{tabular}{|c|c|c|}
\hline Criteria & $\begin{array}{c}\text { Cut-Off Levels for } \\
\text { Differences }\end{array}$ & Control Condition \\
\hline \multicolumn{3}{|l|}{ Gut microbiota activity } \\
\hline Redox potential & $\geq \pm 200 \mathrm{mV}$ & \multirow{5}{*}{$\begin{array}{l}\text { d-Day in treated bioreac- } \\
\text { tor compared to d-Day in } \\
\text { control experiment }\end{array}$} \\
\hline $\mathrm{NaOH}$ consumption & Stop $\mathrm{NaOH}$ consumption & \\
\hline Total gas production & Stop gas production & \\
\hline $\mathrm{CO}_{2}$ concentrations & $\geq \pm 10 \%$ & \\
\hline $\begin{array}{l}\text { SCFA concentrations (acetate, pro- } \\
\text { pionate and butyrate) }\end{array}$ & $\geq \pm 25 \%$ for each SCFA & \\
\hline \multicolumn{3}{|l|}{ Gut microbiota composition } \\
\hline Total viable bacteria-FC & $\geq \pm 1 \log$ & \multirow{2}{*}{$\begin{array}{l}\text { d-Day in treated bioreac- } \\
\text { tor compared to d-Day in } \\
\text { control experiment }\end{array}$} \\
\hline Total bacteria-qPCR & $\geq \pm 1 \log$ & \\
\hline Richness & $\geq \pm 20 \%$ & \multirow{3}{*}{$\begin{array}{l}\text { d-Day compared to day } 6 \\
\text { in treated bioreactor }\end{array}$} \\
\hline Shannon & $\geq \pm 20 \%$ & \\
\hline Bray-Curtis & $\geq 0.5$ & \\
\hline
\end{tabular}

\subsection{Statistical Analysis}

SCFAs (in mM), cytometry, and qPCR data were analyzed using a one-way repeated measure analysis of variance (ANOVA) followed by a Newman-Keuls multiple comparisons test. The statistical analysis was performed using GraphPad Prism software 8.0 (GraphPad Software, Inc., San Diego, CA, USA). Results were expressed as means \pm SEM $(n=3)$. Differences were considered statistically significant when $p<0.05$.

\section{Results}

\subsection{Monitoring of In Vitro Fermentations}

\subsection{1. $\mathrm{NaOH}$ Consumption}

Microbial fermentation activities lead to organic acid production such as SCFA and to a subsequent $\mathrm{pH}$ decrease in the bioreactor, resulting in $\mathrm{NaOH}$ consumption to maintain the $\mathrm{pH}$ at its set point value. Whatever the bioreactor, $\mathrm{NaOH}$ consumption was stable before ATB treatment. Addition of ciprofloxacin led to an immediate interruption in $\mathrm{NaOH}$ consumption that persisted 3 to 4 days after the end of ATB treatment, depending on the experimental runs. FMT treatments led to an earlier restart of $\mathrm{NaOH}$ consumption compared to ATB control, two days before for $30 \mathrm{~g}$ and $10 \mathrm{~g}$ enema and only one day before for oral capsule (in two out of the three replicates, Run3 being similar to ATB control).

\subsubsection{Redox Potential}

Redox potential was also evaluated as an indicator of fermentation activities and anaerobiosis. Before ATB treatment, redox potential stabilized at around $-400 \mathrm{mV}$ in all bioreactors. Addition of ciprofloxacin led to an immediate change with a sharp increase in redox values (up to $0 \mathrm{mV}$ ). At the end of ATB treatment, redox potential slowly decreased to reach baseline values of stabilization phase within 3 to 8 days depending on replicates. Enema treatments (both 30 and $10 \mathrm{~g}$ ) allowed an earlier return to baseline values (except for Run3), four days before ATB control in Run1 and two days before in Run2. For capsule, 
a donor dependent effect was observed with an earlier return to baseline in Run1 (four days before ATB control), no effect in Run2 and a slower return to a stabilized state compared to ATB control in Run3.

\subsection{Gas Production}

At the end of stabilization phase (day 6), gas composition of the atmospheric phase was the same in all tested conditions with approximately $95 \%$ of $\mathrm{CO}_{2}, 4 \% \mathrm{~N}_{2}, 1 \% \mathrm{H}_{2}$, and less than $1 \% \mathrm{O}_{2}$ (Figure 2a-e). This result confirms the ability of maintaining anaerobiosis inside bioreactors without flushing with $\mathrm{CO}_{2}$ or $\mathrm{N}_{2}$ during the total course of fermentation. These relative percentages remained constant throughout control experiments (Figure 2a). As for $\mathrm{NaOH}$ consumption, addition of ciprofloxacin led to an immediate termination in gas overproduction that persisted four days after the end of ATB treatment. This resulted in negative pressure in fermenters that required $\mathrm{N}_{2}$ injection into bioreactors. Moreover, ATB treatment was associated with a change in gas composition (Figure $2 \mathrm{~b}-\mathrm{e}$ ). As illustrated, a sharp increase in relative percentages of $\mathrm{N}_{2}$ was observed due to flushing (from 55 to $80 \%)$ as well as a lower but clear increase in $\mathrm{H}_{2}(1-20 \%)$ and $\mathrm{O}_{2}(5-10 \%)$ that cannot be linked to any gas leak (connection of a gas bag filled with $\mathrm{N}_{2}$ ). Consequently, $\mathrm{CO}_{2}$ relative percentages decreased to $15-20 \%$ (Figure $2 \mathrm{~b}-\mathrm{e}$ ). For ATB control, a return to baseline profiles was observed only 10 days after the end of ciprofloxacin treatment, i.e., at day 22 (Figure 2b). When FMT treatments were applied (Figure 2c-e), gas production restarted faster (1 or 2 days before ATB control) for all modes of administration, except for oral capsule in Run3. Recovery of stabilized profiles (similar to that observed at day 6) was also obtained earlier for 30 and $10 \mathrm{~g}$ enema treatment (day 19) or capsule (day 21) compared to ATB control (day 22). 

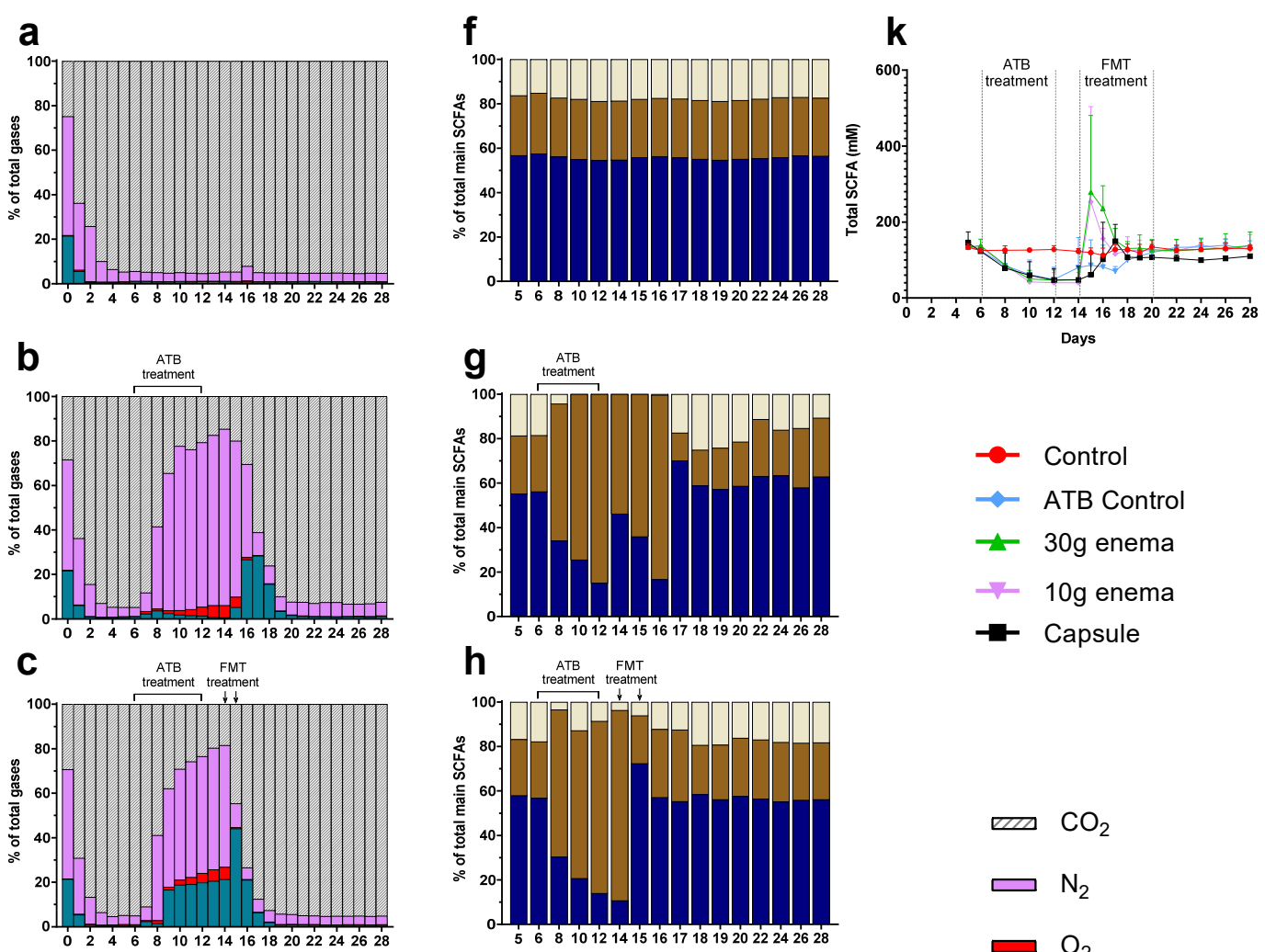

h
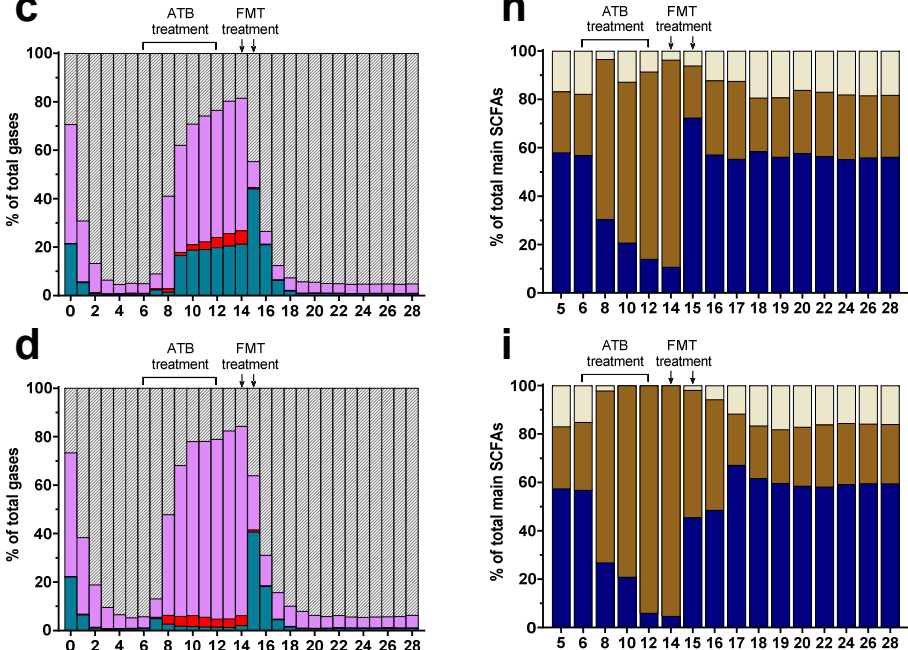

$$
\text { i }
$$

ATB FMT
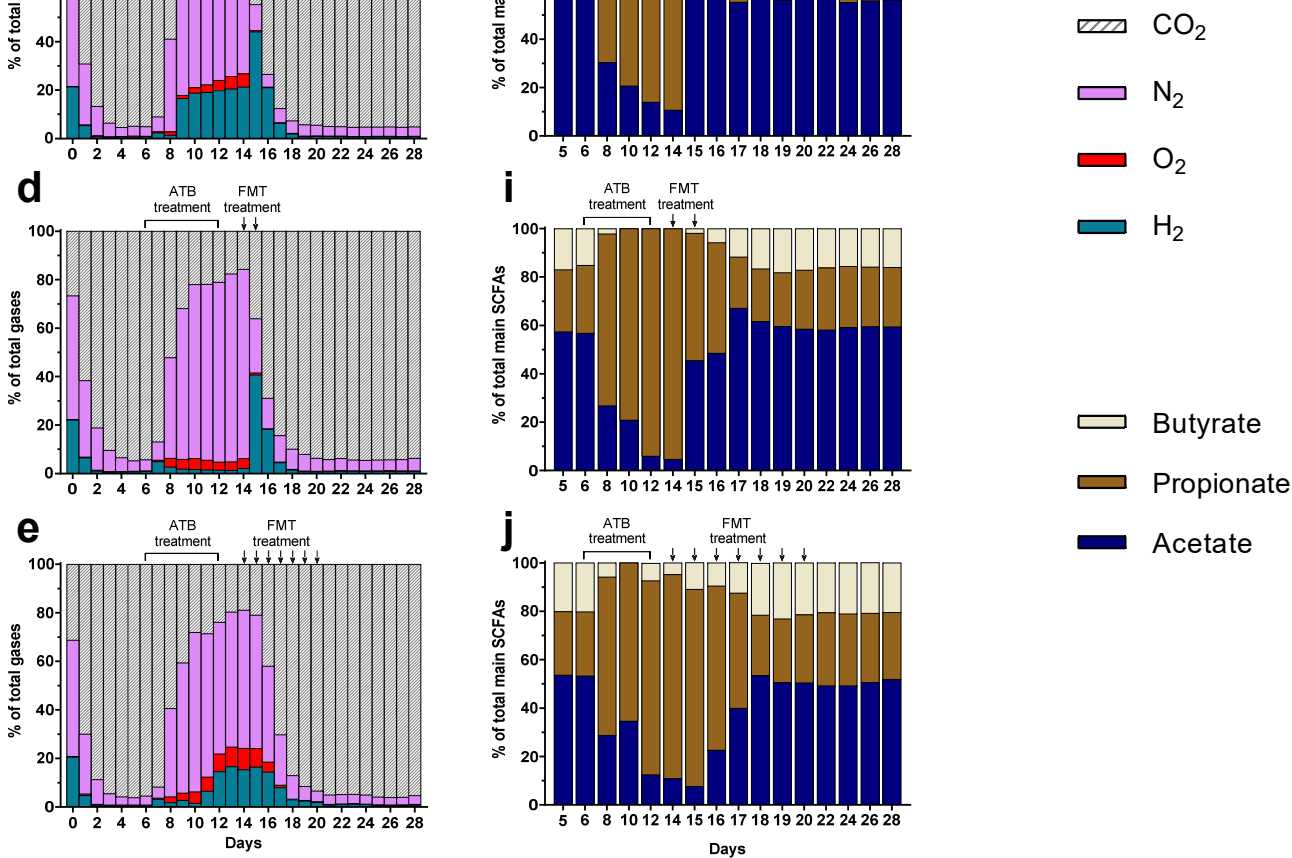

Figure 2. Effect of fecal microbiota transplantation (FMT) treatment on gas and short chain fatty acid (SCFA) production. Experiments were performed as described in Figure 1 and different conditions were applied: no treatment (control, subfigures a and f), ciprofloxacin (ATB control, subfigures b and g), ciprofloxacin and 30 g enema (subfigures $\mathbf{c}$ and $\mathbf{h}$ ), ciprofloxacin and $10 \mathrm{~g}$ enema (subfigures $\mathbf{d}$ and $\mathbf{i}$ ), and ciprofloxacin with oral capsules (subfigures $\mathbf{e}$ and $\mathbf{j}$ ). Gas composition was determined by gas chromatography and results expressed as mean relative percentages (subfigures a to e, $n=3$ ). Main SCFAs (acetate, propionate and butyrate) were analyzed by high performance liquid chromatography. Results were either expressed as mean relative percentages (subfigures $\mathbf{f}$ to $\mathbf{j}, n=3$ ) or as total SCFA concentrations \pm SEM (in $\mathrm{mM}$, subfigure k). 


\subsection{SCFA Production}

Whatever the fermentations, SCFA profiles stabilized at day 6 with relative percentages around $60 \%, 25 \%$, and $15 \%$ for acetate, propionate, and butyrate, respectively (Figure $2 \mathrm{f}-\mathrm{j}$ ) and a total concentration of $130-140 \mathrm{mM}$ (Figure $2 \mathrm{k}$ ). In the control experiment, these percentages remained similar throughout fermentation (Figure 2f). Addition of ciprofloxacin induced a significant decrease $(p<0.05)$ in total SCFA concentrations to reach an approximate concentration of $50 \mathrm{mM}$ at the end of ATB treatment (Figure 2k). ATB treatment also induced changes in SCFAs profiles (Figure $2 \mathrm{~g}-\mathrm{j}$ ) with an important increase in relative percentages of propionate ( $80-95 \%$ at day 12$)$ associated with a decrease in acetate (5$15 \%)$, and butyrate $(0-5 \%)$. These changes persisted at the end of ATB treatment since total SCFA concentrations returned to baseline within 8 days (day 20, Figure 2k). Thus, two additional days (day 22) were needed to recover SCFA proportions similar to those observed at the end of stabilization (Figure 2g). FMT treatment led to a sharp increase in total SCFA concentrations at day 15 for both enema treatments (around $280 \mathrm{mM}$ ), i.e., immediately after the first injection (Figure $2 \mathrm{k}$ ). A donor-dependent response can explain this high variability with a peak at day 15 for Run1, such effect is 4 to 5 times higher than the one observed for Run2 and Run3. Similarly, but to a lesser extent, FMT capsule led to an increase in total SCFA concentrations to reach a maximum of $150 \mathrm{mM}$ at day 17 (Figure $2 \mathrm{k}$ ). All FMT treatments led to a clear reduced time needed to return to stabilized state (24 days before ATB control), with both total SCFA concentrations (Figure $2 \mathrm{k}$ ) and proportions (Figure $2 \mathrm{~h}-\mathrm{j}$ ) similar to baseline values at day 18. Regarding oral capsule, stabilization of total SCFA concentrations occurred at a lower level (100 mM versus 130-140 mM).

\subsection{Quantification of Total Bacteria}

In control experiments, total bacteria stabilized at around 6-7 $\times 10^{9} 16 \mathrm{~S}$ rRNA gene copies/mL, as determined by quantitative PCR analysis (Figure 3a to 3d). Microbial populations were mostly composed of viable cells since a similar population level was obtained by flow cytometry (Figure $3 \mathrm{e}-\mathrm{h}$ ). Total bacteria number and viable bacteria amount were both significantly impacted by ATB treatment with a regular decrease until day 12 in most bioreactors (up to 3-4 logs as demonstrated by qPCR and 2-3 logs as demonstrated by flow cytometry). Nevertheless, the influence of ciprofloxacin impact was not similar in all the experiments, with a minor influence on total and viable bacteria in Run2 for enema $30 \mathrm{~g}$ and capsule conditions (Figure $3 \mathrm{~b}-\mathrm{f}$ ). On average, the return to baseline for ATB control condition occurred progressively within 6 and 7 days after the end of ciprofloxacin administration for total and viable bacteria, respectively (Figure $3 \mathrm{~d}-\mathrm{h}$ ). Likewise, all FMT treatments enable a return of viable bacteria concentrations to stabilized values 3 days before ATB control condition ( $p<0.05$, Figure 3h). Nevertheless, an important variability was observed between the three replicates, more particularly for $30 \mathrm{~g}$ enema and capsule conditions. Especially, the time to recover initial levels in Run3 was similar for FMT capsule when compared to ATB control (Figure 3c-g). 
Total bacteria by qPCR

\section{a}

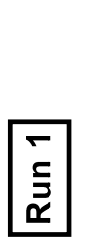

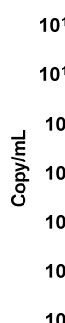

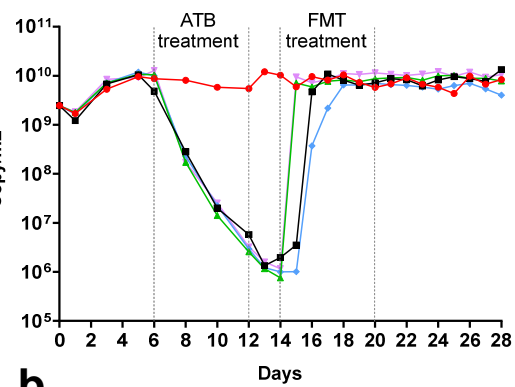

b
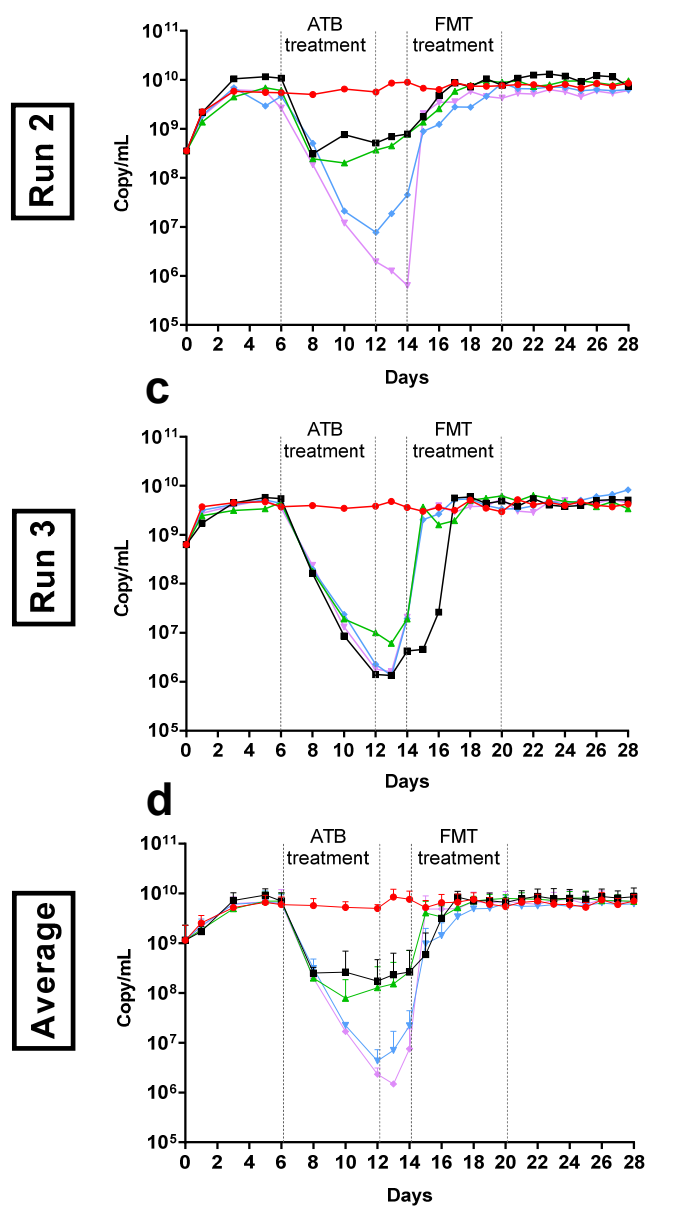

Viable bacteria by cytometry
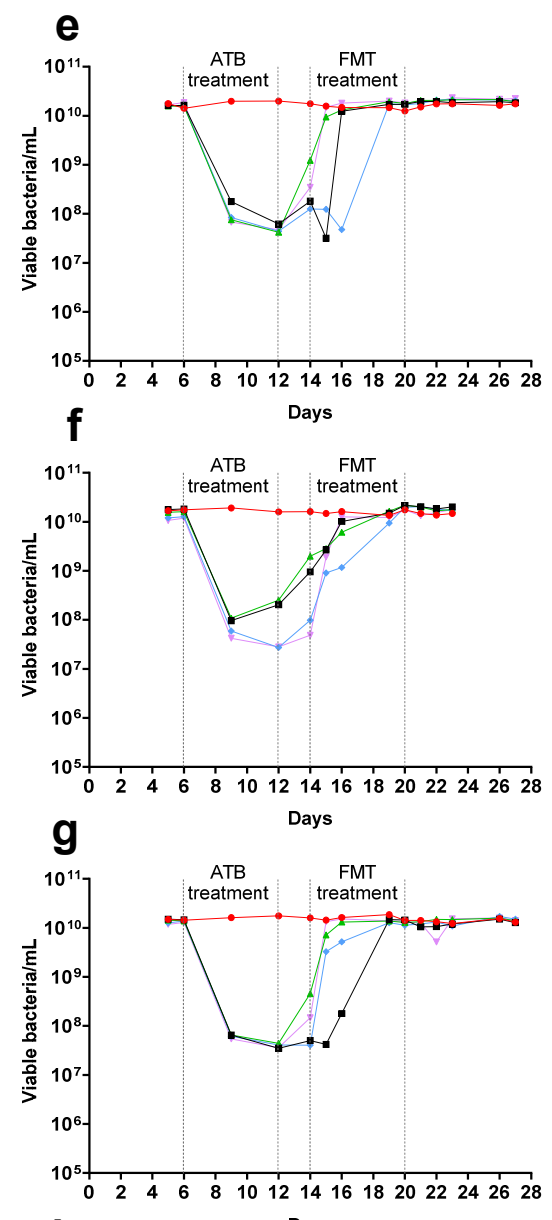

h

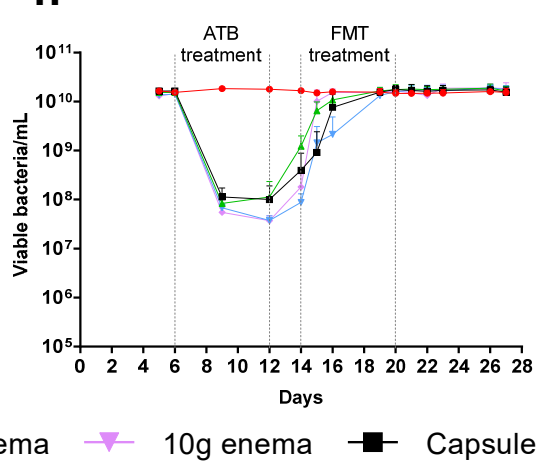

Figure 3. Effect of fecal microbiota transplantation (FMT) treatment on total bacteria. Experiments were performed as described in Figure 1 in triplicate with fecal samples from three healthy adult donors (Run1, Run2 and Run3). Different conditions were applied: no treatment (control, red), ciprofloxacin (ATB control, blue), ciprofloxacin and $30 \mathrm{~g}$ enema (green), ciprofloxacin and $10 \mathrm{~g}$ enema (purple), and ciprofloxacin with oral capsules (black). Total bacteria was determined by qPCR analysis and expressed as numbers of $16 \mathrm{~S}$ rRNA gene copies/mL in Run1 to Run3 (subfigures a to c) or in mean number of copies/mL \pm SEM (subfigure $\mathbf{d}, n=3$ ). Total viable bacteria was determined by flow cytometry through a live/dead analysis and expressed as number of viable cells/mL in Run1 to Run3 (subfigures e to g) or in mean viable cells/mL \pm SEM (subfigure $\mathbf{h}, n=3$ ). 


\subsection{Gut Microbiota Structure}

3.5.1. Composition of Initial Fecal Inoculum and Following Stabilization in ARCOL

Sequencing analysis of the initial fecal suspensions at the phylum level indicated that donor 1 and donor 2 (Supplementary Figure S1, D0, Run1 and 2) exhibited microbial profiles mainly composed of Firmicutes (70\% and 65\%, respectively) and Bacteroidetes (30\% and 35\%, respectively). Donor 3 (Supplementary Figure S1c, D0, Run3) had a reverse Firmicutes/Bacteroidetes ratio (35\%/60\%) with a higher prevalence of Proteobacteria $(3 \%)$. At the family level, dominant taxa of fecal suspensions were as follows: Ruminococcaceae (40\%), Bacteroidaceae (25\%), Lachnospiraceae (20\%), and Veillonellaceae (7\%) for donor 1 (Figure 4, Run 1); Ruminococcaceae (30\%), Prevotellaceae (20\%), Lachnospiraceae (20\%), Bacteroidaceae $(10 \%)$ and Veillonellaceae (10\%), for donor 2 (Figure 4, Run2); and Bacteroidaceae (50\%), Ruminococcaceae (25\%), Lachnospiraceae (10\%), and Rikenellaceae (\%) for donor 3 (Figure 4, Run3).

At the end of stabilization phase in ARCOL (Supplementary Figure S1, day 6) phyla profiles were quite similar for all runs with a large majority of Bacteroidetes $(60-70 \%)$, followed by Firmicutes (20-40\%), and Proteobacteria (1-3\%). At the family level (Figure 4), profiles at day 6 appear to be run-dependent with close composition for Run1 and Run3 while being clearly different for Run2. Run1 and Run3 exhibited a high abundance of Bacteroidaceae ( $70-80 \%$ and $65-75 \%$, respectively) followed by Ruminococcaceae ( $6 \%$ and $15 \%$, respectively) and Lachnospiraceae ( $5 \%$ and $10 \%$, respectively). Gut microbiota in Run1 also displayed $4 \%$ of Veillonellaceae. For Run2, profiles are composed of stable abundances of Ruminococcaceae (30-40\%), Lachnospiraceae (10-15\%), and Veillonellaceae (1-3\%) while Prevotellaceae and Bacteroidaceae were more variable between conditions (from 2 to $40 \%$ ). Stabilized profiles remain almost constant for all control experiments during the 28 days of fermentation (Figure 4). 

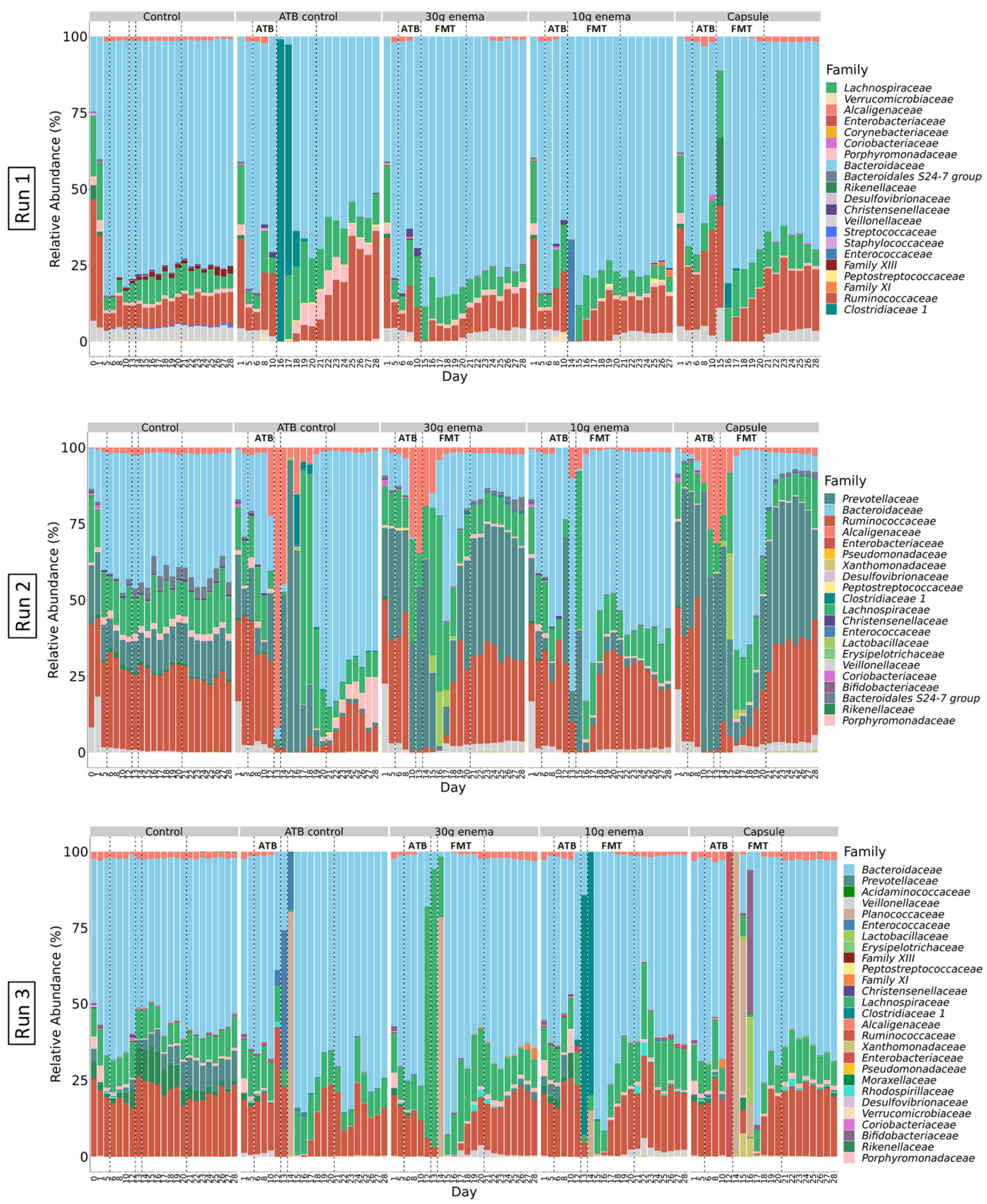

Figure 4. Effect of fecal microbiota transplantation (FMT) treatment on microbial composition at family level.

Experiments were performed as described in Figure 1 in triplicate with fecal samples from three healthy adult donors (Run1, Run2 and Run3). Different conditions were applied: no treatment (control), ciprofloxacin (ATB control), ciprofloxacin and $30 \mathrm{~g}$ enema, ciprofloxacin and $10 \mathrm{~g}$ enema, and ciprofloxacin with oral capsules. Microbial composition was determined at the family level by $16 \mathrm{~S}$ rRNA gene sequencing and expressed as relative abundances. 


\subsubsection{Impact of ATB Treatment}

Addition of ciprofloxacin led to pronounced changes in microbial profiles even at the phylum level. For Run1 and Run3, at the phylum level, major changes were characterized by an increase in Firmicutes abundance and a disappearance of Proteobacteria (Supplementary Figure S1). In Run1 only, Verrucomicrobia (up to 3\%) appeared during ATB treatment. For Run2, opposite trends were observed with a decrease in Firmicutes abundance associated with a sharp increase in Proteobacteria (up to $90 \%$ in ATB control). At the family level, ATB treatment had also a strong influence on microbial structure with variations between experimental runs and even between bioreactors for a single run. Major changes for Run1 were represented by an increase in Ruminococcaceae and Lachnospiraceae together with a loss of Veillonellaceae and Porphyromonodaceae (Figure 4). For Run2, Prevotellaceae and Alcaligenaceae abundances were mostly increased while those of Bacteroidaceae and Porphyromonodaceae declined. Lastly for Run3, according to bioreactors, the main variations observed were a bloom of one or several families among the Enterococcaceae, Planococcaceae, Clostridiaceae or Lachnospiraceae. Perturbations of microbial profiles persisted following cessation of ATB treatment in control experiments (ATB control). Stabilization occurred only around day 25-27 but with different profiles when compared to day 6, both at phylum (Supplementary Figure S1) and family (Figure 4) levels.

\subsubsection{Effect of FMT Treatments}

All FMT treatments induced a rapid and clear shift (from day 15) in microbial profiles both at phylum (Supplementary Figure S1) and family levels (Figure 4). Microbial abundances kept evolving after this initial shift until 5-8 days after the first FMT administration to reach a new stable profile close from the one observed at the end of stabilization phase. Some taxa, such as Veillonellaceae in all runs, Bacteroidales S24-7 group in Run2 and Alcaligenaceae and Porphyromonodaceae in Run3, which disappeared during ATB treatment, have been shown to reappear in FMT-treated bioreactors only (but not in ATB control). On the contrary, some families present in control experiments, such as Prevotellaceae in Run3, were no longer present after ATB treatment even in FMT-treated bioreactors (Figure 4). Interestingly, in Run2, Prevotella 7, the main genus from Prevotellaceae, present at the end of stabilization phase was substituted by Paraprevotella during ATB treatment which disappeared again in favor of Prevotella 7 when capsule and $30 \mathrm{~g}$ enema treatments were applied.

\subsection{Microbial Richness and Diversity}

\subsection{1. $\alpha$ - Diversity}

Alpha-diversity was first evaluated by calculating sample richness at the OTU level, i.e., the number of different OTUs reflecting species in a sample (Figure 5, panel A). In control experiments, for all three donors (Run1, Run2 and Run3), richness stabilized at around 150-200 OTUs all along the fermentation process. Administration of ciprofloxacin led to a rapid and pronounced decrease of richness index to reach less than 10 OTUs at the end of ATB treatment. At the end of ciprofloxacin injection in ATB control experiments, richness increased to stabilize at day 18-20, but at lower values when compared to control assays (around 80-100 OTUs). Interestingly, FMT treatment enable a return of richness values to baseline levels within 6-8 days after the end of ATB treatment. Shannon index was also calculated to better picture diversity and species distribution in the various samples (Supplementary Figure S2). In control experiments, Shannon index stabilized at values around 2-3 from day 6 to day 28. ATB treatment was associated with a sharp decrease of Shannon index, with value below 1 . For ATB control, Shannon index regularly increased to reach baseline levels the last 2 days of experiments, with an exception for Run3 where stabilized values remained lower than the one at day 6 (around 2 versus 3). When FMT treatments were performed, Shannon index recovered baseline values within 
2 to 6 days after the first administration for enema formulae and after 6 days for oral capsule.

A
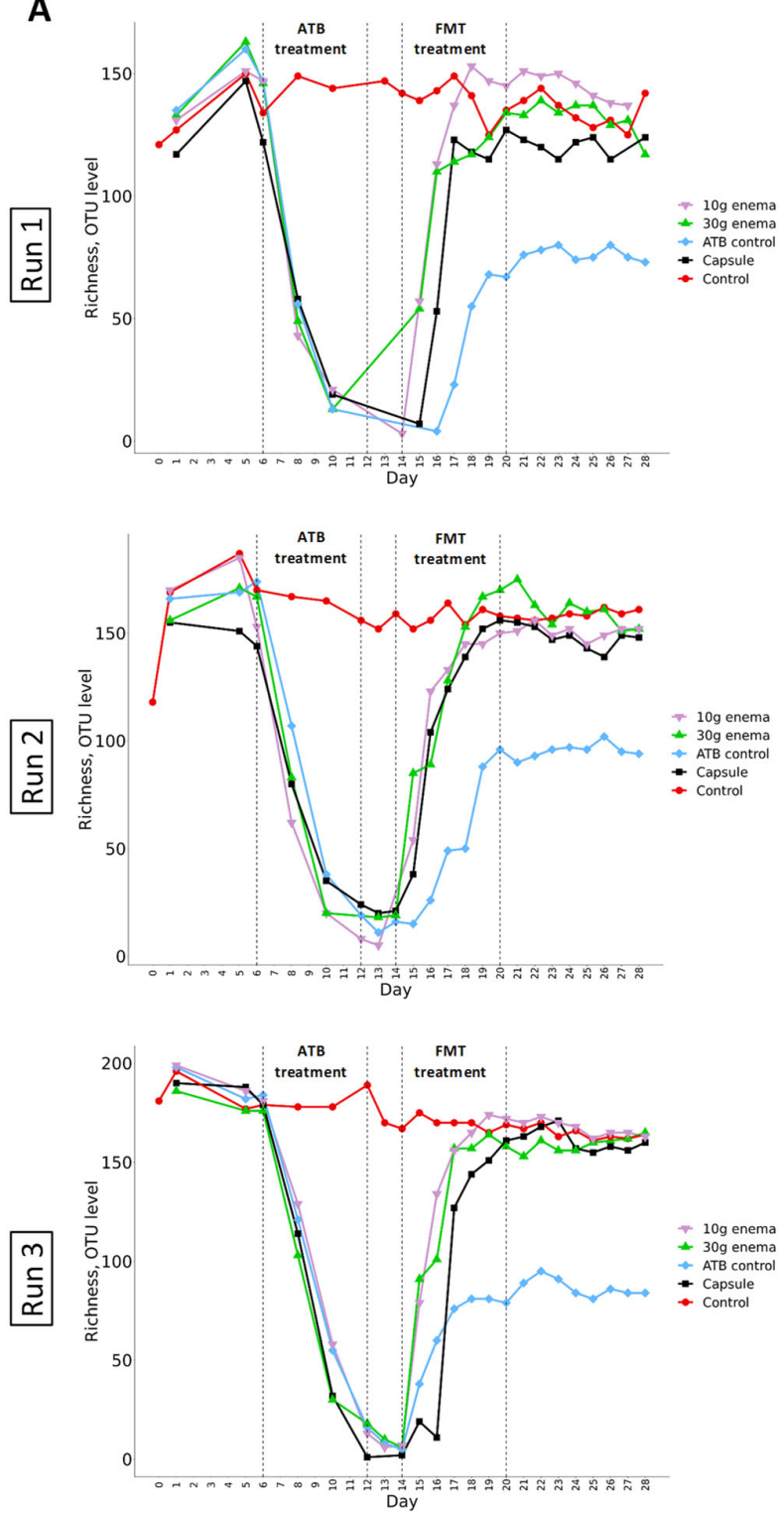

B
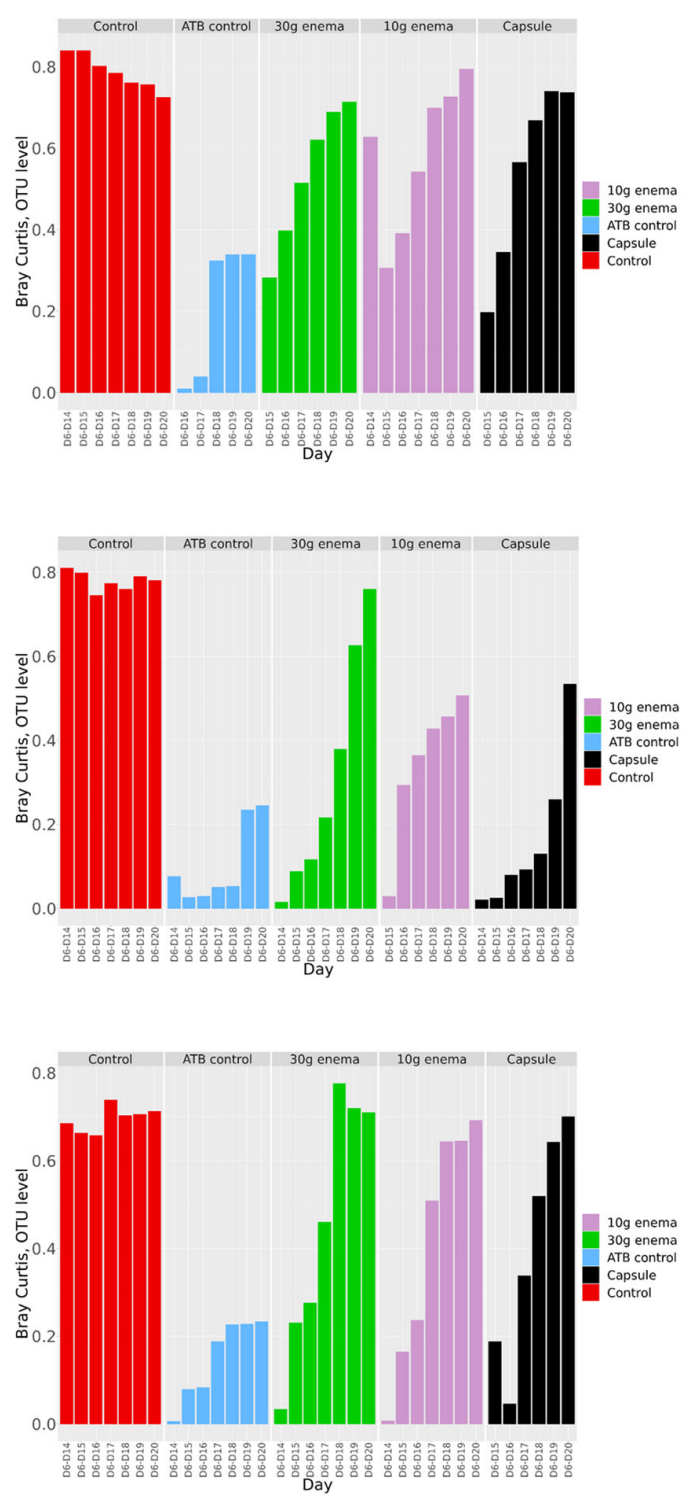

Figure 5. Effect of fecal microbiota transplantation (FMT) treatment on richness and Bray-Curtis indexes at OTU level.

Experiments were performed as described in Figure 1 in triplicate with fecal samples from three healthy adult donors (Run1, Run2 and Run3). Different conditions were applied: no treatment (control, red), ciprofloxacin (ATB control, blue), ciprofloxacin and 30 g enema (green), ciprofloxacin and $10 \mathrm{~g}$ enema (purple), and ciprofloxacin with oral capsules (black). Richness (panel A) and Bray-Curtis (panel B, compared to day 6 values) indexes were determined at the OTU level after 16S-rRNA gene sequencing. Bray-Curtis values represent similarity of samples versus day 6 .

\subsection{2. $\beta$ - Diversity}

Bray-Curtis dissimilarity was calculated as an indicator of $\beta$-diversity compared to values obtained at day 6 (end of stabilization phase for each bioreactor). Results obtained for the three replicates Run1, Run2 and Run3 are presented in Figure 5 panel B. Whatever 
the replicates, similar trends were observed. For control experiments, Bray-Curtis similarity index versus day 6 remained stable during fermentations (except for Run3 where a slight decrease was noted). When no FMT treatment was applied to bioreactor following ciprofloxacin administration (ATB control), a clear shift from control experiments was observed with values being much lower (from 0.2 to 0.3 ). Nonetheless, whatever the replicates (Run1, Run2 or Run3), FMT treatment induced a clear restoration of microbial diversity since all samples progressively normalize to control values, except for $10 \mathrm{~g}$ enema and oral capsule in Run2 (indexes at around 0.5 at the end of fermentation). For all other tested conditions, return to baseline values was observed between day 18 and day 20 for all FMT modes of administration.

\subsection{Determination of Dysbiotic Periods}

In order to assess FMT efficiency in restoring gut microbiota composition and activity, as well as the influence of mode of administration, the number of "dysbiotic days" was determined. This number of "dysbiotic days" was calculated for each variable listed in Table 2 and for each tested condition (ATB control, $30 \mathrm{~g}$ enema, $10 \mathrm{~g}$ enema, and capsule). Results were expressed as average of the three replicates (Supplementary Table S1) and represented in Figure 6. These results confirmed that ATB control experiments exhibited the highest number of dysbiotic days with an average of 12.1 days (Supplementary Table S1). FMT treatment clearly decreased dysbiotic periods with a similar value of 7.6 days for both $30 \mathrm{~g}$ enema and $10 \mathrm{~g}$ enema. For oral capsule, a longer dysbiotic period of 8.3 days was observed (non-significant). When analyzing results in depth especially at microbiota structure and activity levels, different situations were observed depending on FMT mode of administration. Regarding microbial activity, capsule was the less efficient technique with 9.4 days of dysbiosis, followed by $10 \mathrm{~g}$ enema ( 8.7 days), while $30 \mathrm{~g}$ enema showed the best score (7.7 days). This difference was mainly due to a high number of dysbiotic days for capsule in relation with acetate production (Figure 6) and probably results from a latent period required for microbial revivification of capsule freeze-dried form. Regarding microbial structure, different ranking was observed, with $30 \mathrm{~g}$ enema being the less efficient (7.6 days) followed by capsule ( 6.9 days) and $10 \mathrm{~g}$ enema (6.1 days). The lower efficiency of $30 \mathrm{~g}$ enema resulted from a higher number of dysbiotic days in relation with all the microbial diversity indexes. 


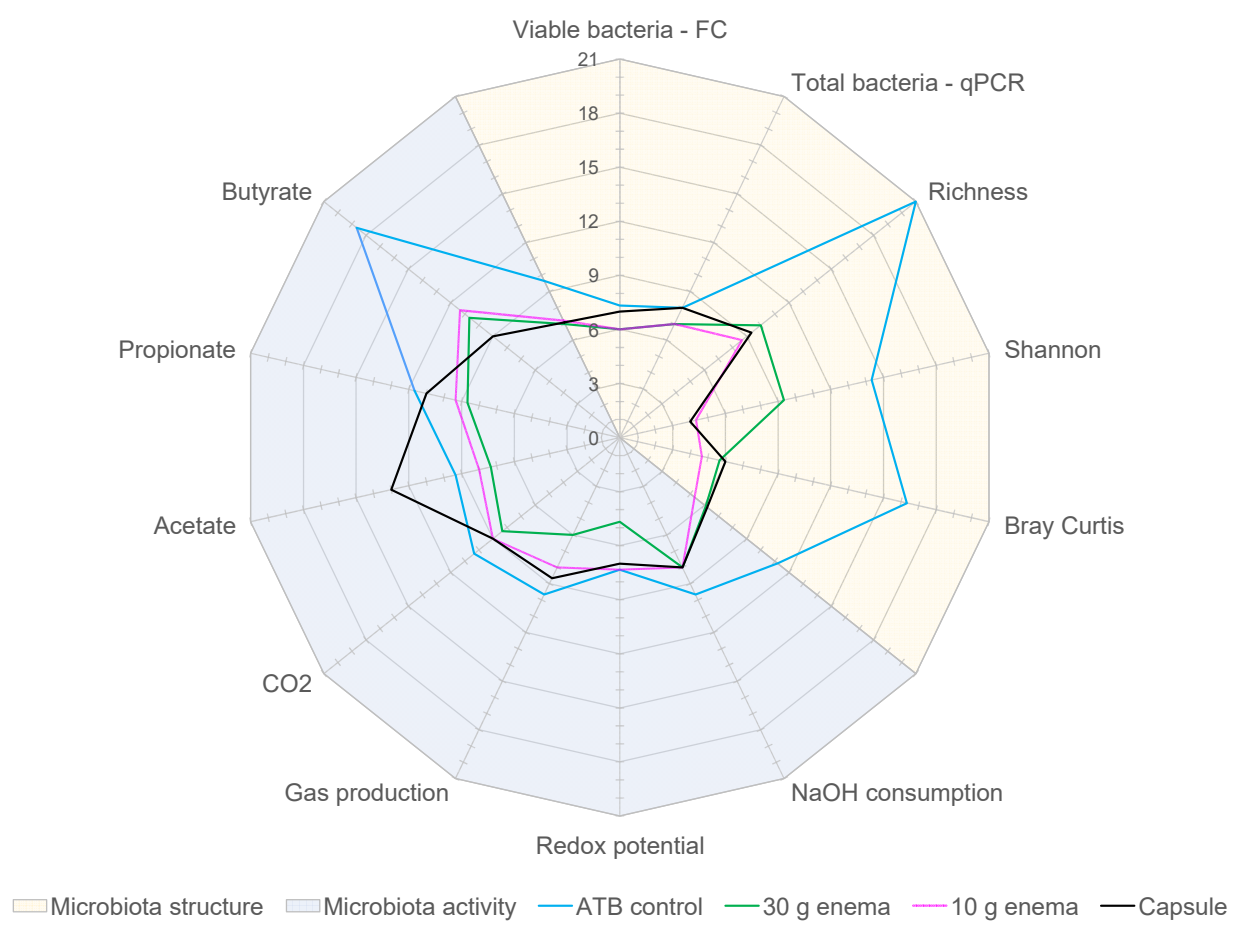

Figure 6. Effect of fecal microbiota transplantation (FMT) treatment on the duration of dysbiosis.

Experiments were performed as described in Figure 1 and different conditions were applied: ciprofloxacin (ATB control, blue), ciprofloxacin and 30 g enema (green), ciprofloxacin and $10 \mathrm{~g}$ enema (purple), and ciprofloxacin with oral capsules (black). For each tested condition, the number of dysbiotic days was determined and compared to the appropriate control using criteria described in Table 2 (in relation with both gut microbiota structure and activity) and expressed as mean number of dysbiotic days $(\mathrm{n}=3)$.

\section{Discussion}

In this study, we described for the first time the use of a dynamic computer-controlled in vitro model of the human colon, namely ARCOL, as an alternative to in vivo assays in rodents for testing different FMT formulations following antibiotic treatment. The in vitro model was challenged with ciprofloxacin, an antibiotic widely used in humans, to reproduce clinically relevant gut microbiota perturbations. Then, the "dysbiotic" model was efficiently used to evaluate different modes of FMT administration. This study demonstrates that a new oral capsule was as efficient as more traditional invasive enemas to restore gut microbiota structure and activity and to decrease the number of disturbed days.

The first aim of the present study was to establish a medically relevant "dysbiotic" in vitro model of the human gut ecosystem. For this purpose, we used the in vitro colon model ARCOL, set-up to mimic, based on in vivo data, not only physicochemical parameters of the adult human colon but also microbial components [27-30,41]. This model was previously validated under healthy conditions, i.e., with a non-disturbed microbiota. As the drastic effect of antibiotics on human gut microbiota is well acknowledged by the scientific community [42], we decided to apply an antibiotic treatment to induce gut microbiota perturbations in ARCOL system, as already performed in other colon models $[25,26,43]$. Ciprofloxacin, a fluoroquinolone antibiotic widely used in hospital settings to treat various digestive and extra-digestive bacterial infections, was chosen in the present 
study [44]. This antibiotic was also selected due to available information on its pharmacokinetic in humans, especially on its fecal excretion [45]. The dose $(500 \mu \mathrm{g} / \mathrm{mL})$ and the mode of administration (first initial input followed by a continuous supply for 7 days) were established considering hospital practices, ciprofloxacin metabolism (percentage of absorption in the human upper gut and estimated fecal antibiotic clearance) and in vitro colon parameters (total volume and retention time). Ciprofloxacin concentrations were checked by antibiotic dosage throughout ARCOL experiments $(500 \mu \mathrm{g} / \mathrm{mL})$. Our results clearly indicated that ciprofloxacin induced in vitro rapid changes in gut microbial composition and activity that heightened during antibiotic administration. These modifications clearly include a reduction of overall microbial richness and diversity and a loss in microbial fermentation capacities, in accordance with in vivo data in humans, even if available data have not been obtained exactly under the same operational conditions (e.g., dose) $[46,47]$. In addition, in some replicates, a rise in dominance of bacterial species usually subdominant, including pathobionts such as Enterobacteriaceae and some Clostridiaceae, was observed. All these changes are indicative of a gut microbiota dysbiosis state in accordance with human data [4,5]. Interestingly, at the family level, effects of ciprofloxacin were widely donor dependent in ARCOL, as previously reported in humans and mice [48]. In the current in vitro study, we describe for the first time an original index that quantitates the dysbiosis state in ARCOL. This dysbiosis index was calculated based on the total number of dysbiotic days in bioreactors. To consider all aspects related to dysbiosis definition [4,5], this index integrates criteria related to both microbial structure (bacterial population relative abundances, microbial richness and diversity) and functions (related to main fermentation products such as gases and SCFA).

Once the "dysbiotic" in vitro colon model was established and validated according to in vivo data in humans from literature, we evaluated the ability of various FMT formulations to restore the balance of gut microbial communities in ARCOL model. Three different forms were tested, i.e., two dosages of enema and one oral capsule. Until 1990, enema was the method of choice for FMT and still remains frequently used in hospitals [14]. Enema is less invasive, easier to perform and relatively less expensive than colonoscopy or upper gastro-intestinal routes, even if the approach can be limited by patient compliance and difficulty to retain the suspension. Two doses were tested, $30 \mathrm{~g}$ enema which is commonly used in clinical practices and $10 \mathrm{~g}$ enema to comparatively assess the efficiency of a lower microbial quantity. The effect of these two formulations was compared to that of a new caecum-releasing capsule containing a freeze-dried form of the enema formulation. Oral capsule is the most recently developed mode of stool delivery, the first formulation being described in 2014 [49,50]. Capsules which are esthetically pleasant, convenient, and minimally invasive are preferred by patients and are advantageously the cheapest FMT mode of administration $[19,49,50]$. To be as close as possible to clinical procedure, enemas were directly introduced into ARCOL model, since in humans they are delivered into the distal colon via a cannula. The oral capsule tested in this study was specifically designed to open and release its content at the end of human small intestine only. In a preliminary pilot study, we evaluated capsule integrity in ileal effluents of the human TNO gastrointestinal, TIM, system by visual control and bacterial numeration [14]. As the capsules remained intact until the end of small intestine in vitro, capsule content was directly added as a suspension in ARCOL system in the present work.

Our data demonstrated that all tested forms were able to accelerate return to a stable state compared to ATB control, thereby reinforcing the resilience of the ecosystem. FMT treatments had an immediate impact on gut microbiota structure while there was a latency for recovery of a stable microbial activity. This phenomenon was due to necessary adaptation of newly added bacteria. Interestingly, the lowest enema dosage (10 g) was as efficient as the highest one $(30 \mathrm{~g})$ and reduced in a similar way the number of dysbiotic days (of approximately 4.5 days). To our knowledge, no previous published report had already investigated a dose effect for FMT enema in vitro. These in vitro results suggest that bac- 
terial load may be reduced without any deleterious effect while restoring microbial balance after antibiotic-induced dysbiosis. The oral capsule proved almost as efficient as enema forms (-3.8 dysbiotic days), despite a lower amount of administered bacteria (100 times lower) and a longer administration period (1 week compared to two days). In previous studies, two approaches were mainly developed for FMT oral capsules, first freezing at $-80^{\circ} \mathrm{C}$ with glycerol and more recently freeze-drying with various cryoprotectants, as performed in the present study [50-52]. Interestingly, Jiang and colleagues showed in mice that there was no difference in efficiency between frozen and lyophilized capsules in rCDI treatment. Moreover, the authors demonstrated that products can be stored up to 7 months without losing gut microbiota composition and therapeutic efficacy [51]. Oral capsules were also designed for delivery in various luminal segments of the gastro-intestinal tract. A comparative study between two capsules preparations, with either gastric or colonic delivery properties, showed that the colonic-release form tended to be the most effective in rCDI, particularly in restoring Bacteroidetes phylum and increasing gut microbial diversity [50]. This result is in accordance with the strategy promoted in the current study testing a caecum-release oral formulation. To date, few studies in humans have already compared the efficiency of oral capsules (frozen or lyophilized) to more traditional FMT routes (such as colonoscopy or enema) for the treatment of rCDI. In accordance with our results, despite the differences of tested formulations from other studies and ours, available data in humans indicate that oral capsules are as effective as traditional modes of administration, especially to restore bacterial diversity [19,52]. Allegretti and colleagues have shown in humans that the lower dose treatment with colonic release capsules (10 capsules in a single administration) was equally effective to cure rCDI than the higher treatment-dose (30 capsules) [50]. This is fully in accordance with our results on enema forms, showing the relevance of in vitro approach for FMT evaluation.

This study confirms the potential of in vitro gut models to mimic gut microbiota dysbiotic state and for subsequent assessment of FMT efficiency. Such approach appears as a relevant alternative to in vivo animal assays in preclinical phases. Interestingly, in vitro results could appropriately document technical files ahead of clinical trials in humans. Although they accurately report the ecological dynamics in terms of structure and function, these models are hindered by their inability to fully reproduce the overall processes occurring in vivo, especially hormonal and nervous controls, feedback mechanisms, local immune system and host-bacterial mutualism. Particularly, patients receiving FMT are often subjected to diverse external stresses that cannot be integrated in vitro but may widely influence gut physiology and microbiota restoration. Yet, for obvious regulatory, ethics and cost reasons, in vitro colon models are advantageous over in vivo assays due to their flexibility, accuracy and reproducibility [53]. This approach is also adequately in line with the European and North American 3R rules aiming to minimize the number of animals used for research purpose and promote the development of alternative in vitro methods. As a stable microbiome can be maintained in bioreactors over a long timeframe, the effect of successive treatments can also be tested. In addition, gut models can be adapted to perform colon-segment specific research $[31,54]$ and several bioreactors can be inoculated with the same fecal sample to perform in parallel control and treatment experiments. Lastly, as shown here, inter-individual gut microbiota variability can be considered by performing replicates with fecal samples from different donors. In the present study, ARCOL was inoculated with stools from healthy adult donors but we can envision to extend its potentialities (as previously performed in other human colon models) by using fecal samples from selected age groups, such as infants [55-57] or elderly subjects [58,59], or from patients suffering from cancers, inflammatory bowel diseases or metabolic disorders [60-63]. To be fully relevant, a complete adaptation of physicochemical parameters of the colon ( $\mathrm{pH}$, transit time, composition of nutritive medium and biliary salts) to the specific targeted population would be required. This would unlock new applications in human health through in vitro evaluation of FMT potential to restore gut microbiota eubiosis in these pathological situations. 
To conclude, this study describes for the first time the use of an in vitro human colon model, adapted to reproduce clinically relevant perturbations by antibiotherapy, for the evaluation of various FMT formulations. By integrating the main physicochemical parameters of the human colon ( $\mathrm{pH}$, retention time, nutrient supply and anaerobiosis), ARCOL model was shown to capture microbial diversity and inter-individual variability of the human gut microbiome. Treatment with ciprofloxacin led to a marked state of dysbiosis with a sharp decrease in fermentation activities, a loss of microbial diversity and a shift in bacterial populations, as previously described in vivo in humans. The three formulations tested for autologous FMT, i.e., $30 \mathrm{~g}$ enema, $10 \mathrm{~g}$ enema and a new caecum-release oral capsule, showed similar effects by clearly reducing the time to restore gut microbiota structure and activity. Due to regulatory, ethic, cost and technical advantages, in vitro gut models such as ARCOL can be advantageously used in preclinical phases as an alternative to in vivo assays in animals. Their potential in human health may be extended to the evaluation of allogenic FMT and could help in selecting the best clinical protocols (e.g., period for treatment, frequency and duration of FMT administration) when targeting various age groups and intestinal or extra-digestive pathologies.

Supplementary Materials: The following are available online at www.mdpi.com/xxx, Figure S1: Effect of FMT treatment on microbial composition at phylum level, Figure S2: Effect of FMT treatment on Shannon index at OTU level, Table S1: Effect of FMT treatment on the duration of dysbiosis state (number of dysbiotic days) in the ARCOL model.

Author Contributions: C.V. and S.D. did laboratory work, performed sampling and analyzed the data. C.V. and O.U. wrote the first draft of the manuscript. S.B.-D. designed the study and provided support for data analysis and writing the manuscript. D.D., C.L.C., J.D. and C.S. assisted in the study design and provided constructive feedbacks on the manuscript. C.G. and L.B. performed sequencing and bioinformatics analysis. All authors have read and agreed to the published version of the manuscript.

Funding: This study was funded by Maat Pharma company.

Institutional Review Board Statement: Not applicable. This study being a non-interventional study with no addition to usual clinical care, according to the French Health Public Law (CSP Art L 11211.1), the protocol does not require approval from an ethic committee.

Informed Consent Statement: Informed consent was obtained from all subjects involved in the study.

Data availability statement: All sequencing data were deposited to the National Center for Biotechnology Information Sequence Read Archive under accession number PRJNA642894.

Conflicts of Interest: No potential conflicts of interest were disclosed.

\section{References}

1. Eckburg, P.B.; Bik, E.M.; Bernstein, C.N.; Purdom, E.; Dethlefsen, L.; Sargent, M.; Gill, S.R.; Nelson, K.E.; Relman, D.A. Diversity of the Human Intestinal Microbial Flora. Science 2005, 308, 1635-1638, doi:10.1126/science.1110591.

2. Hugon, P.; Lagier, J.-C.; Colson, P.; Bittar, F.; Raoult, D. Repertoire of Human Gut Microbes. Microb. Pathog. 2017, 106, 103-112, doi:10.1016/j.micpath.2016.06.020.

3. Walker, A.W.; Duncan, S.H.; Louis, P.; Flint, H.J. Phylogeny, Culturing, and Metagenomics of the Human Gut Microbiota. Trends Microbiol. 2014, 22, 267-274, doi:10.1016/j.tim.2014.03.001.

4. Malard, F.; Gasc, C.; Plantamura, E.; Doré, J. High Gastrointestinal Microbial Diversity and Clinical Outcome in Graft-versusHost Disease Patients. Bone Marrow Transplant. 2018, 53, 1493-1497, doi:10.1038/s41409-018-0254-x.

5. Vangay, P.; Ward, T.; Gerber, J.S.; Knights, D. Antibiotics, Pediatric Dysbiosis, and Disease. Cell Host Microbe 2015, 17, 553-564, doi:10.1016/j.chom.2015.04.006.

6. Zhang, F.; Cui, B.; He, X.; Nie, Y.; Wu, K.; Fan, D.; FMT-Standardization Study Group. Microbiota Transplantation: Concept, Methodology and Strategy for Its Modernization. Protein Cell 2018, 9, 462-473, doi:10.1007/s13238-018-0541-8.

7. Wang, J.-W.; Kuo, C.-H.; Kuo, F.-C.; Wang, Y.-K.; Hsu, W.-H.; Yu, F.-J.; Hu, H.-M.; Hsu, P.-I.; Wang, J.-Y.; Wu, D.-C. Fecal Microbiota Transplantation: Review and Update. J. Formos. Med. Assoc. 2019, 118, S23-S31, doi:10.1016/j.jfma.2018.08.011.

8. Eiseman, B.; Silen, W.; Bascom, G.S.; Kauvar, A.J. Fecal Enema as an Adjunct in the Treatment of Pseudomembranous Enterocolitis. Surgery 1958, 44, 854-859. 
9. Khan, M.Y.; Dirweesh, A.; Khurshid, T.; Siddiqui, W.J. Comparing Fecal Microbiota Transplantation to Standard-of-Care Treatment for Recurrent Clostridium Difficile Infection: A Systematic Review and Meta-Analysis. Eur. J. Gastroenterol. Hepatol. 2018, 30, 1309-1317, doi:10.1097/MEG.0000000000001243.

10. DuPont, H.L.; Jiang, Z.-D.; DuPont, A.W.; Utay, N.S. Abnormal Intestinal Microbiome in Medical Disorders and Potential Reversibility by Fecal Microbiota Transplantation. Dig. Dis. Sci. 2020, 65, 741-756, doi:10.1007/s10620-020-06102-y.

11. Gupta, S.; Allen-Vercoe, E.; Petrof, E.O. Fecal Microbiota Transplantation: In Perspective. Ther. Adv. Gastroenterol. 2016, 9, 229239, doi:10.1177/1756283X15607414.

12. Ooijevaar, R.E.; Terveer, E.M.; Verspaget, H.W.; Kuijper, E.J.; Keller, J.J. Clinical Application and Potential of Fecal Microbiota Transplantation. Annu. Rev. Med. 2019, 70, 335-351, doi:10.1146/annurev-med-111717-122956.

13. DeFilipp, Z.; Hohmann, E.; Jenq, R.R.; Chen, Y.-B. Fecal Microbiota Transplantation: Restoring the Injured Microbiome after Allogeneic Hematopoietic Cell Transplantation. Biol. Blood Marrow Transplant. 2019, 25, e17-e22, doi:10.1016/j.bbmt.2018.10.022.

14. Ramai, D.; Zakhia, K.; Ofosu, A.; Ofori, E.; Reddy, M. Fecal Microbiota Transplantation: Donor Relation, Fresh or Frozen, Delivery Methods, Cost-Effectiveness. Ann. Gastroenterol. 2019, 32, 30-38, doi:10.20524/aog.2018.0328.

15. Taur, Y.; Coyte, K.; Schluter, J.; Robilotti, E.; Figueroa, C.; Gjonbalaj, M.; Littmann, E.R.; Ling, L.; Miller, L.; Gyaltshen, Y.; et al. Reconstitution of the Gut Microbiota of Antibiotic-Treated Patients by Autologous Fecal Microbiota Transplant. Sci. Transl. Med. 2018, 10, doi:10.1126/scitranslmed.aap9489.

16. Kelly, C.R.; Khoruts, A.; Staley, C.; Sadowsky, M.J.; Abd, M.; Alani, M.; Bakow, B.; Curran, P.; McKenney, J.; Tisch, A.; et al. Effect of Fecal Microbiota Transplantation on Recurrence in Multiply Recurrent Clostridium Difficile Infection: A Randomized Trial. Ann. Intern. Med. 2016, 165, 609-616, doi:10.7326/M16-0271.

17. Heath, R.D.; Cockerell, C.; Mankoo, R.; Ibdah, J.A.; Tahan, V. Fecal Microbiota Transplantation and Its Potential Therapeutic Uses in Gastrointestinal Disorders. North. Clin. Istanb. 2018, 5, 79-88, doi:10.14744/nci.2017.10692.

18. Kim, K.O.; Gluck, M. Fecal Microbiota Transplantation: An Update on Clinical Practice. Clin. Endosc. 2019, 52, 137-143, doi:10.5946/ce.2019.009.

19. Kao, D.; Roach, B.; Silva, M.; Beck, P.; Rioux, K.; Kaplan, G.G.; Chang, H.-J.; Coward, S.; Goodman, K.J.; Xu, H.; et al. Effect of Oral Capsule- vs Colonoscopy-Delivered Fecal Microbiota Transplantation on Recurrent Clostridium Difficile Infection: A Randomized Clinical Trial. JAMA 2017, 318, 1985-1993, doi:10.1001/jama.2017.17077.

20. Le Bastard, Q.; Ward, T.; Sidiropoulos, D.; Hillmann, B.M.; Chun, C.L.; Sadowsky, M.J.; Knights, D.; Montassier, E. Fecal Microbiota Transplantation Reverses Antibiotic and Chemotherapy-Induced Gut Dysbiosis in Mice. Sci. Rep. 2018, 8, 6219, doi:10.1038/s41598-018-24342-x.

21. Wei, Y.-L.; Chen, Y.-Q.; Gong, H.; Li, N.; Wu, K.-Q.; Hu, W.; Wang, B.; Liu, K.-J.; Wen, L.-Z.; Xiao, X.; et al. Fecal Microbiota Transplantation Ameliorates Experimentally Induced Colitis in Mice by Upregulating AhR. Front. Microbiol. 2018, 9, 1921, doi:10.3389/fmicb.2018.01921.

22. Wrzosek, L.; Ciocan, D.; Borentain, P.; Spatz, M.; Puchois, V.; Hugot, C.; Ferrere, G.; Mayeur, C.; Perlemuter, G.; Cassard, A.-M. Transplantation of Human Microbiota into Conventional Mice Durably Reshapes the Gut Microbiota. Sci. Rep. 2018, 8, 6854, doi:10.1038/s41598-018-25300-3.

23. Zhou, J.; Zhou, Z.; Ji, P.; Ma, M.; Guo, J.; Jiang, S. Effect of Fecal Microbiota Transplantation on Experimental Colitis in Mice. Exp. Ther. Med. 2019, 17, 2581-2586, doi:10.3892/etm.2019.7263.

24. Hartung, T. Thoughts on Limitations of Animal Models. Parkinsonism Relat. Disord. 2008, 14, S81-S83, doi:10.1016/j.parkreldis.2008.04.003.

25. McDonald, J.A.K.; Mullish, B.H.; Pechlivanis, A.; Liu, Z.; Brignardello, J.; Kao, D.; Holmes, E.; Li, J.V.; Clarke, T.B.; Thursz, M.R.; et al. Inhibiting Growth of Clostridioides Difficile by Restoring Valerate, Produced by the Intestinal Microbiota. Gastroenterology 2018, 155, 1495-1507, doi:10.1053/j.gastro.2018.07.014.

26. Moura, I.B.; Normington, C.; Ewin, D.; Clark, E.; Wilcox, M.H.; Buckley, A.M.; Chilton, C.H. Method Comparison for the Direct Enumeration of Bacterial Species Using a Chemostat Model of the Human Colon. BMC Microbiol. 2020, 20, doi:10.1186/s12866019-1669-2.

27. Blanquet-Diot, S.; Denis, S.; Chalancon, S.; Chaira, F.; Cardot, J.-M.; Alric, M. Use of Artificial Digestive Systems to Investigate the Biopharmaceutical Factors Influencing the Survival of Probiotic Yeast during Gastrointestinal Transit in Humans. Pharm. Res. 2012, 29, 1444-1453, doi:10.1007/s11095-011-0620-5.

28. Thévenot, J.; Etienne-Mesmin, L.; Denis, S.; Chalancon, S.; Alric, M.; Livrelli, V.; Blanquet-Diot, S. Enterohemorrhagic Escherichia Coli O157:H7 Survival in an in Vitro Model of the Human Large Intestine and Interactions with Probiotic Yeasts and Resident Microbiota. Appl. Environ. Microbiol. 2013, 79, 1058-1064, doi:10.1128/AEM.03303-12.

29. Thévenot, J.; Cordonnier, C.; Rougeron, A.; Le Goff, O.; Nguyen, H.T.T.; Denis, S.; Alric, M.; Livrelli, V.; Blanquet-Diot, S. Enterohemorrhagic Escherichia Coli Infection Has Donor-Dependent Effect on Human Gut Microbiota and May Be Antagonized by Probiotic Yeast during Interaction with Peyer's Patches. Appl. Microbiol. Biotechnol. 2015, 99, 9097-9110, doi:10.1007/s00253-015-6704-0.

30. Deschamps, C.; Fournier, E.; Uriot, O.; Lajoie, F.; Verdier, C.; Comtet-Marre, S.; Thomas, M.; Kapel, N.; Cherbuy, C.; Alric, M.; et al. Comparative Methods for Fecal Sample Storage to Preserve Gut Microbial Structure and Function in an in Vitro Model of the Human Colon. Appl. Microbiol. Biotechnol. 2020, 104, 10233-10247, doi:10.1007/s00253-020-10959-4. 
31. Van den Abbeele, P.; Grootaert, C.; Marzorati, M.; Possemiers, S.; Verstraete, W.; Gérard, P.; Rabot, S.; Bruneau, A.; El Aidy, S.; Derrien, M.; et al. Microbial Community Development in a Dynamic Gut Model Is Reproducible, Colon Region Specific, and Selective for Bacteroidetes and Clostridium Cluster IX. Appl. Environ. Microbiol. 2010, 76, 5237-5246, doi:10.1128/AEM.00759-10.

32. Hösl, J.; Gessner, A.; El-Najjar, N. Liquid Chromatography-Tandem Mass Spectrometry for the Quantification of Moxifloxacin, Ciprofloxacin, Daptomycin, Caspofungin, and Isavuconazole in Human Plasma. J. Pharm. Biomed. Anal. 2018, 157, 92-99, doi:10.1016/j.jpba.2018.05.015.

33. Lefeuvre, S.; Bois-Maublanc, J.; Hocqueloux, L.; Bret, L.; Francia, T.; Eleout-Da Violante, C.; Billaud, E.M.; Barbier, F.; Got, L. A Simple Ultra-High-Performance Liquid Chromatography-High Resolution Mass Spectrometry Assay for the Simultaneous Quantification of 15 Antibiotics in Plasma. J. Chromatogr. B 2017, 1065-1066, 50-58, doi:10.1016/j.jchromb.2017.09.014.

34. Yu, Y.; Lee, C.; Kim, J.; Hwang, S. Group-Specific Primer and Probe Sets to Detect Methanogenic Communities Using Quantitative Real-Time Polymerase Chain Reaction. Biotechnol. Bioeng. 2005, 89, 670-679, doi:10.1002/bit.20347.

35. Magoč, T.; Salzberg, S.L. FLASH: Fast Length Adjustment of Short Reads to Improve Genome Assemblies. Bioinformatics 2011, 27, 2957-2963, doi:10.1093/bioinformatics/btr507.

36. Bolger, A.M.; Lohse, M.; Usadel, B. Trimmomatic: A Flexible Trimmer for Illumina Sequence Data. Bioinformatics 2014, 30, 21142120, doi:10.1093/bioinformatics/btu170.

37. Langmead, B.; Salzberg, S.L. Fast Gapped-Read Alignment with Bowtie 2. Nat. Methods 2012, 9, 357-359, doi:10.1038/nmeth.1923.

38. Rognes, T.; Flouri, T.; Nichols, B.; Quince, C.; Mahé, F. VSEARCH: A Versatile Open Source Tool for Metagenomics. PeerJ 2016, 4, e2584, doi:10.7717/peerj.2584.

39. Silva. SSU Database Release 128. Available online: https://www.arb-silva.de/documentation/release-128/ (accessed on 25 May 2020).

40. R. The R Project for Statistical Computing. Available online: https://www.r-project.org/ (accessed on 25 May 2020).

41. Cordonnier, C.; Thévenot, J.; Etienne-Mesmin, L.; Denis, S.; Alric, M.; Livrelli, V.; Blanquet-Diot, S. Dynamic In Vitro Models of the Human Gastrointestinal Tract as Relevant Tools to Assess the Survival of Probiotic Strains and Their Interactions with Gut Microbiota. Microorganisms 2015, 3, 725-745, doi:10.3390/microorganisms3040725.

42. Iizumi, T.; Battaglia, T.; Ruiz, V.; Perez Perez, G.I. Gut Microbiome and Antibiotics. Arch. Med. Res. 2017, 48, 727-734, doi:10.1016/j.arcmed.2017.11.004.

43. Auchtung, J.M.; Robinson, C.D.; Britton, R.A. Cultivation of Stable, Reproducible Microbial Communities from Different Fecal Donors Using Minibioreactor Arrays (MBRAs). Microbiome 2015, 3, doi:10.1186/s40168-015-0106-5.

44. Campoli-Richards, D.M.; Monk, J.P.; Price, A.; Benfield, P.; Todd, P.A.; Ward, A. Ciprofloxacin. A Review of Its Antibacterial Activity, Pharmacokinetic Properties and Therapeutic Use. Drugs 1988, 35, 373-447, doi:10.2165/00003495-198835040-00003.

45. Krueger, W.A.; Ruckdeschel, G.; Unertl, K. Influence of Intravenously Administered Ciprofloxacin on Aerobic Intestinal Microflora and Fecal Drug Levels When Administered Simultaneously with Sucralfate. Antimicrob. Agents Chemother. 1997, 41, 1725-1730.

46. Lange, K.; Buerger, M.; Stallmach, A.; Bruns, T. Effects of Antibiotics on Gut Microbiota. Dig. Dis. 2016, 34, 260-268, doi:10.1159/000443360.

47. Willmann, M.; Vehreschild, M.J.G.T.; Biehl, L.M.; Vogel, W.; Dörfel, D.; Hamprecht, A.; Seifert, H.; Autenrieth, I.B.; Peter, S. Distinct Impact of Antibiotics on the Gut Microbiome and Resistome: A Longitudinal Multicenter Cohort Study. BMC Biol. 2019, 17, 76, doi:10.1186/s12915-019-0692-y.

48. Zimmermann, P.; Curtis, N. The Effect of Antibiotics on the Composition of the Intestinal Microbiota - A Systematic Review. J. Infect. 2019, 79, 471-489, doi:10.1016/j.jinf.2019.10.008.

49. Youngster, I.; Russell, G.H.; Pindar, C.; Ziv-Baran, T.; Sauk, J.; Hohmann, E.L. Oral, Capsulized, Frozen Fecal Microbiota Transplantation for Relapsing Clostridium Difficile Infection. JAMA 2014, 312, 1772-1778, doi:10.1001/jama.2014.13875.

50. Allegretti, J.R.; Fischer, M.; Sagi, S.V.; Bohm, M.E.; Fadda, H.M.; Ranmal, S.R.; Budree, S.; Basit, A.W.; Glettig, D.L.; de la Serna, E.L.; et al. Fecal Microbiota Transplantation Capsules with Targeted Colonic Versus Gastric Delivery in Recurrent Clostridium Difficile Infection: A Comparative Cohort Analysis of High and Lose Dose. Dig. Dis. Sci. 2019, 64, 1672-1678, doi:10.1007/s10620-018-5396-6.

51. Jiang, Z.-D.; Alexander, A.; Ke, S.; Valilis, E.M.; Hu, S.; Li, B.; DuPont, H.L. Stability and Efficacy of Frozen and Lyophilized Fecal Microbiota Transplant (FMT) Product in a Mouse Model of Clostridium Difficile Infection (CDI). Anaerobe 2017, 48, 110114, doi:10.1016/j.anaerobe.2017.08.003.

52. Jiang, Z.-D.; Jenq, R.R.; Ajami, N.J.; Petrosino, J.F.; Alexander, A.A.; Ke, S.; Iqbal, T.; DuPont, A.W.; Muldrew, K.; Shi, Y.; et al. Safety and Preliminary Efficacy of Orally Administered Lyophilized Fecal Microbiota Product Compared with Frozen Product given by Enema for Recurrent Clostridium Difficile Infection: A Randomized Clinical Trial. PLoS ONE 2018, 13, e0205064, doi:10.1371/journal.pone.0205064.

53. Payne, A.N.; Zihler, A.; Chassard, C.; Lacroix, C. Advances and Perspectives in in Vitro Human Gut Fermentation Modeling. Trends Biotechnol. 2012, 30, 17-25, doi:10.1016/j.tibtech.2011.06.011.

54. Fehlbaum, S.; Chassard, C.; Poeker, S.A.; Derrien, M.; Fourmestraux, C.; Lacroix, C. Clostridium Difficile Colonization and Antibiotics Response in PolyFermS Continuous Model Mimicking Elderly Intestinal Fermentation. Gut Pathogens 2016, 8, 63, doi:10.1186/s13099-016-0144-y. 
55. Davidovics, Z.H.; Michail, S.; Nicholson, M.R.; Kociolek, L.K.; Pai, N.; Hansen, R.; Schwerd, T.; Maspons, A.; Shamir, R.; Szajewska, H.; et al. Fecal Microbiota Transplantation for Recurrent Clostridium Difficile Infection and Other Conditions in Children: A Joint Position Paper From the North American Society for Pediatric Gastroenterology, Hepatology, and Nutrition and the European Society for Pediatric Gastroenterology, Hepatology, and Nutrition. J. Pediatr. Gastroenterol. Nutr. 2019, 68, 130-143, doi:10.1097/MPG.0000000000002205.

56. Gurram, B.; Sue, P.K. Fecal Microbiota Transplantation in Children: Current Concepts. Curr. Opin. Pediatr. 2019, 31, 623-629, doi:10.1097/MOP.0000000000000787.

57. Pham, V.T.; Chassard, C.; Rifa, E.; Braegger, C.; Geirnaert, A.; Rocha Martin, V.N.; Lacroix, C. Lactate Metabolism Is Strongly Modulated by Fecal Inoculum, PH, and Retention Time in PolyFermS Continuous Colonic Fermentation Models Mimicking Young Infant Proximal Colon. mSystems 2019, 4, doi:10.1128/mSystems.00264-18.

58. Fehlbaum, S.; Chassard, C.; Haug, M.C.; Fourmestraux, C.; Derrien, M.; Lacroix, C. Design and Investigation of PolyFermS In Vitro Continuous Fermentation Models Inoculated with Immobilized Fecal Microbiota Mimicking the Elderly Colon. PLoS ONE 2015, 10, e0142793, doi:10.1371/journal.pone.0142793.

59. Cheng, Y.-W.; Fischer, M. The Present Status of Fecal Microbiota Transplantation and Its Value in the Elderly. Curr. Treat. Options Gastroenterol. 2017, 15, 349-362, doi:10.1007/s11938-017-0143-1.

60. Vermeiren, J.; Van den Abbeele, P.; Laukens, D.; Vigsnaes, L.K.; De Vos, M.; Boon, N.; Van de Wiele, T. Decreased Colonization of Fecal Clostridium Coccoides/Eubacterium Rectale Species from Ulcerative Colitis Patients in an in Vitro Dynamic Gut Model with Mucin Environment. FEMS Microbiol. Ecol. 2012, 79, 685-696, doi:10.1111/j.1574-6941.2011.01252.x.

61. Bussolo de Souza, C.; Roeselers, G.; Troost, F.; Jonkers, D.; Koenen, M.E.; Venema, K. Prebiotic Effects of Cassava Bagasse in TNO's in Vitro Model of the Colon in Lean versus Obese Microbiota. J. Funct. Foods 2014, 11, 210-220, doi:10.1016/j.jff.2014.09.019.

62. Dai, Z.; Zhang, J.; Wu, Q.; Fang, H.; Shi, C.; Li, Z.; Lin, C.; Tang, D.; Wang, D. Intestinal Microbiota: A New Force in Cancer Immunotherapy. Cell Commun. Signal. 2020, 18, doi:10.1186/s12964-020-00599-6.

63. Wang, Y.; Ma, W.; Abu-Sbeih, H.; Jiang, Z.-D.; DuPont, H.L. Fecal Microbiota Transplantation (FMT) for Immune Checkpoint Inhibitor Induced-Colitis (IMC) Refractory to Immunosuppressive Therapy. JCO 2020, 38, 3067-3067, doi:10.1200/JCO.2020.38.15_suppl.3067. 